\title{
The excitation within the molecular hydrogen jets of the protostellar outflow $\mathrm{HH} 212^{\star}$
}

\author{
M. D. Smith ${ }^{1,2}$, B. O’Connell ${ }^{1,3}$, and C. J. Davis ${ }^{4}$ \\ 1 Armagh Observatory, College Hill, Armagh BT61 9DG, Northern Ireland, UK \\ e-mail: m.d.smith@kent.ac.uk, boc@arm.ac.uk \\ 2 Centre for Astrophysics \& Planetary Science, The University of Kent, Canterbury CT2 7NH, UK \\ 3 Physics Department, Trinity College Dublin, College Green, Dublin 2, Ireland \\ 4 Joint Astronomy Centre, 660 N.A'ohoku Place, University Park, Hilo, Hawaii 96720, USA \\ e-mail: c.davis@jach.hawaii.edu
}

Received 10 November 2006 / Accepted 30 January 2007

\begin{abstract}
The near-infrared twin jets emanating from the HH 212-mm protostar are remarkable for their symmetry. By performing integral field spectroscopy with the UIST imager-spectrometer on UKIRT, we investigate the chains of bright knots and arcs within the jets to gain insight into the underlying physics and dynamics. We obtain numerous images associated with line emission from vibrationallyexcited molecular hydrogen and the [Fe II] line at $1.64 \mu \mathrm{m}$. This allows us to study the spatial variation in excitation of the inner knots and outer bow-shaped objects. We find that the excitation properties are consistent with outward-moving bow shocks close to the plane of the sky. However, there is a gradient in excitation transverse to the jet axis across the inner knots on the scale of 0.1 arcsec. This C-shaped inner symmetry suggests a transverse source motion rather than precession, possibly related to the jet bending and the transverse gradient in radial velocity. Moreover, the bow models predict that the iron emission should peak further ahead of the molecular emission than actually observed. This leads us to propose that each inner knot consists of two closely-spaced asymmetric bows, as found for the outer bows which clearly occur in distinct pairs, well-separated in a lower density environment. The weak inter-knot emission may then be generated within oblique shock waves resulting from the deflection of fluid across asymmetric bow flanks.
\end{abstract}

Key words. ISM: jets and outflows - circumstellar matter - infrared: ISM - Herbig-Haro objects - stars: formation

\section{Introduction}

The near-infrared outflow $\mathrm{HH} 212$ is powered by a protostar, HH 212-mm, concealed within a cool condensation, IRAS 05413-0104. The $K$-band emission is dominated by lines of molecular hydrogen with the 1-0 S(1) transition at $2.12 \mu \mathrm{m}$ producing spectacular images (Zinnecker et al. 1998). The highly collimated outflow contains two prominent inner knots which appeared point-like in the initial low resolution observations, at first suggesting an interpretation as a young binary system (Reipurth 1989; Zinnecker et al. 1992). However, deeper observations revealed that the two bright points are the first pair in a series of knots and bow shocks which delineate the outflow (Zinnecker et al. 1996, 1998). Here, we present images of the bright features taken in a range of near-infrared lines and consider how these structures are formed. The entire $\mathrm{H}_{2}$ outflow and the features we study are displayed in Fig. 1.

IRAS 05413-0104 is located in the L1630 molecular cloud about $90^{\prime}$ north-east of the Horsehead nebula at a distance of $\sim 460 \mathrm{pc}$. The protostar has been detected at 25, 60 and $100 \mu \mathrm{m}$ but is too cool and embedded to be detected as yet at $12 \mu \mathrm{m}$ (Beichman et al. 1986). It is a low-mass protostar with a bolometric luminosity estimated to be about $8 L_{\odot}$ and a bolometric temperature of $\approx 56 \mathrm{~K}$ (Froebrich et al. 2003). The ratio of

* Tables 1-3 are only available in electronic form at http: //www . aanda.org submillimetre to bolometric luminosity is about 0.009 , which bestows the Class 0 classification (e.g. Froebrich 2005).

The outflow itself covers an extent of $\sim 240^{\prime \prime}$, or $0.54 \mathrm{pc}$. The total $\mathrm{H}_{2}$ 1-0 S(1) luminosity is $6.9 \times 10^{-3} L_{\odot}$ (not corrected for extinction) (Stanke 2000). A series of inner knots, which are regularly spaced with inter-knot distances of $\sim 4$ " or $\sim 2000 \mathrm{AU}$, extend on both sides of the bipolar $\mathrm{H}_{2}$ outflow. Emission is also detected in the inter-knot spaces: the knots appear to be connected by a thin stream of emission (Zinnecker et al. 1998; McCaughrean et al. 2002). Both series of knots terminate in small bow shocks at a distance of about 13000 AU from the central position (NB1 \& SB1). These bow shocks are composed of two separate arcs nested together. Whereas the brightness of the knots decreases with distance from the source (Zinnecker et al. 1998; McCaughrean et al. 2002), the bow shocks break the trend and appear relatively bright again. This could partly be due to a reduction in the extinction as the outflow breaks free of its maternal envelope. Other significant features are as follows.

- The outflow is inclined within a few degrees of the plane of the sky. This is deduced from a small difference in radial velocity between the inner knots of $\sim 10 \mathrm{~km} \mathrm{~s}^{-1}$ (Zinnecker et al. 1998; Davis et al. 2000) which implies a flow just $2^{\circ}$ from the sky plane on assuming a jet speed of $\sim 120 \mathrm{~km} \mathrm{~s}^{-1}$. In addition, the inner $\mathrm{H}_{2}$ emission is equally bright on each side, suggesting that the inner outflow has equal intrinsic brightness and extinction on both sides. The emission from the larger outer bows is not equally bright which would 


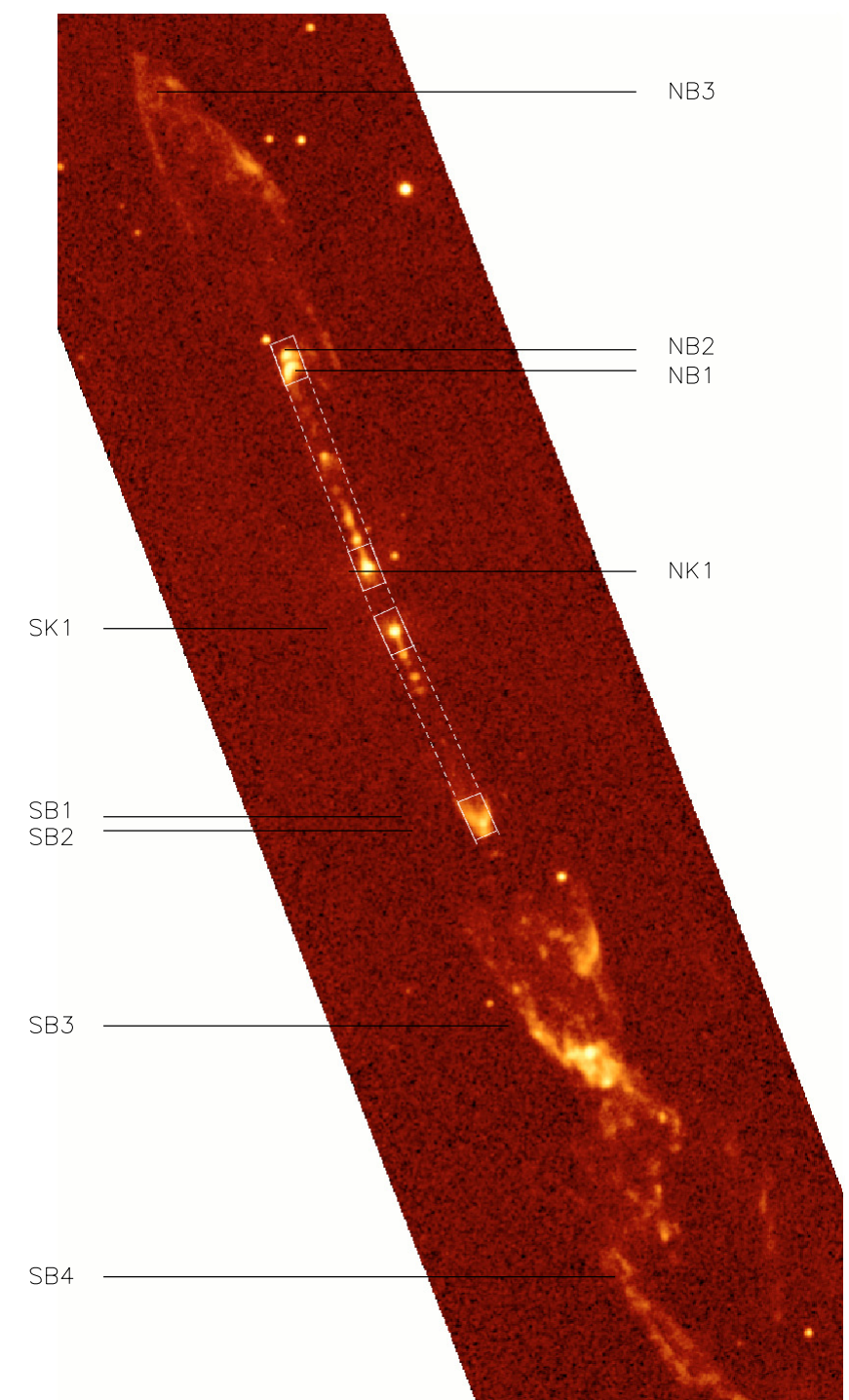

Fig. 1. The entire HH212 outflow at $2.122 \mu \mathrm{m}$, displaying the line emission from the $\mathrm{H}_{2}$ 1-0 S(1) transition. The image was obtained at the Calar Alto 3.5-m telescope by Zinnecker et al. (1998). The outflow covers an extent of $240^{\prime \prime}$ (54 pc). The IRAS source lies between the two bright knots NK 1 and SK 1 with central position RA $(2000)=$ $05^{\mathrm{h}} 43^{\mathrm{m}} 51.4^{\mathrm{s}}, \operatorname{Dec}(2000)=-01^{\circ} 02^{\prime} 52^{\prime \prime}$. The locations of the effective apertures of the IFU observations are superimposed.

imply that the external medium is no longer evenly distributed at this distance from the source. Indeed, the giant southern bow SB4 has no northern counterpart.

- The southern and northern jet directions deviate by $\sim 2^{\circ}$ from being anti-parallel. This is consistent with a motion of the driving source relative to the cloud core (by a few $\mathrm{km} \mathrm{s}^{-1}$ ) or a motion of the cloud core relative to the external cloud medium (ram pressure). Alternatively, the jet direction could be influenced by the orbital motion of the jet source within a young binary system (Fendt \& Zinnecker 1998, 2000).

- The outer bows SB 3 and NB 3 are wide and open and show $\mathrm{H}_{2}$ line emission spread from the bow apices into the extended bow wings. A cluster of background galaxies appears near bow NB 3, suggesting that there must be very little ambient material surrounding the bow, i.e. it is a sharp dense feature.

- The apices of the giant outer bows are slightly displaced from their jet axes and in opposite directions. In addition, the outermost southern $\mathrm{H}_{2}$ bows SB 3 and SB 4 exhibit a morphology reminiscent of a helical structure. This serves as tentative evidence of directional variability and rotation of the jet during early ejection events.

- A pair of diffuse nebulae divided by a dark lane is detected at the base of the jet in the $K$-band. The nebulae are almost exclusively $\mathrm{H}_{2}$ line emission (McCaughrean et al. 2002) implying that the source of illumination is not the protostar itself. One likely explanation is that the bright inner knots provide the line emission which is scattered from the outer surface of the flattened rotating cloud core surrounding the protostar.

- Deep optical images have failed to detect emission from $\mathrm{H} \alpha$ and [S II] (Zinnecker et al. 1996). This is not due to extinction for the outer bows but, as the near-infrared data here will show, the inner knots lie behind considerable extinction (see Sect. 4.2). Instead, HH 212 may well be an almost purely molecular jet.

- A smooth velocity gradient of about $4-5 \mathrm{~km} \mathrm{~s}^{-1} \mathrm{pc}^{-1} \mathrm{ex}-$ ists across the flattened disk-like $\mathrm{NH}_{3}$ core (Wiseman et al. 2001), suggestive of east-west rotation around an axis aligned with the jet. Transverse velocity gradients were also detected across some of the knots (Davis et al. 2000). Although not conclusive, the sense of the gradient is consistent with the $\mathrm{NH}_{3}$ disk, suggesting that rotation is responsible.

- A systematic increase in radial velocity of the inner $\mathrm{H}_{2}$ knots (NK 1-NK 7 and SK 1-SK 3) with distance from the source is found (Davis et al. 2000). The northern jet shows increasingly red-shifted radial velocities whereas the southern jet shows increasing blue-shifted velocities. Such a shift in radial velocities could be caused by jet acceleration or by an S-shaped bending of the jet of order a few degrees every $10000 \mathrm{AU}$. Such a bending is compatible with the noncollinearity of the jet seen in the plane of the sky.

- Emission in the [Fe II] line is strong from the inner knots and at a much lower level from NB 1, SB 1 and NK 7 (Zinnecker et al. 1998; McCaughrean et al. 2002).

- $\mathrm{SiO}$ emission close to the central source as well as along the southern jet (at a position $20^{\prime \prime}$ from the source) has been detected (Gibb et al. 2004). The detection of strong SiO emission in the jet is indicative of the Class $\mathrm{O}$ nature of the outflow, as discussed by Gibb et al. (2004).

- CO $J=1-0$ line emission is detected close to the $\mathrm{H}_{2}$ bow shocks, suggesting that the $\mathrm{CO}$ emission traces material which is being entrained by jet-driven bow shocks (Lee et al. 2000). Furthermore, as revealed in position-velocity diagrams, the broadest range in $\mathrm{CO}$ radial velocities is found to be coincident with the tips of the bow shocks (Lee et al. 2000), as was also found for HH 240/241 (O'Connell et al. 2004).

We selected the bright inner pair of knots and the most prominent pair of bows for a detailed spectroscopic study. We performed similar excitation imaging studies for other well-defined chains of $\mathrm{H}_{2}$ knots and bows belonging to the flows HH 7-11 (Khanzadyan et al. 2003), HH 240 (O'Connell et al. 2004) and HH 211 (O'Connell et al. 2005). We found evidence that supersonic ambipolar diffusion, in which an ion-magneto gas diffuses through a neutral gas, is in operation. In this process, molecules are collisionally excited by the streaming ions over an extended region. The result is a continuous $\mathrm{C}$-type shock. In addition, continuous near-infrared emission occurs in HH 211 and atomic line emission is associated with the caps of bow shocks and other radiative J-type shock structures. 
The Integral Field Unit (IFU) described below delivers near infrared images at various wavelengths simultaneously. Consequently, changes in atmospheric transmission or seeing have no influence on relative knot morphologies or line ratios, and accurate subtraction of the continuum is possible. Moreover, the relative positions of the emission peaks for various transition lines of $\mathrm{H}_{2}$ and [Fe II] (at $1.644 \mu \mathrm{m}$ ) can be determined without the need for registration using field stars. These observations and the data reduction techniques are described in Sect. 2 and the results presented in Sect. 3. In Sect. 4, the results are analysed and a bow shock model is presented.

\section{Observations and data reduction}

The United Kingdom Infra-Red Telescope (UKIRT) $1.5 \mu \mathrm{m}$ Imager Spectrometer UIST is designed to switch quickly between imaging and spectroscopy modes. It is equipped with a $1024 \times 1024 \mathrm{InSb}$ array. In spectroscopy mode, the internal optics yield $0.12^{\prime \prime}$ per pixel. UIST is capable of spectroscopy over a two dimensional field of view using the Integral Field Unit (IFU). The IFU covers a $3.3^{\prime \prime} \times 6.0^{\prime \prime}$ (rotatable) sky area with a plate scale of $0.24^{\prime \prime} \times 0.12^{\prime \prime}$. An image-slicing mirror divides the incident image into 18 adjacent 0.24 " $\times 6.0$ " "slitlets", of which 14 are usable. The emission through each slitlet is directed onto a dispersing grism and fed through the UIST optical system as if it were a single long-slit, the 14 spectral images from the useable slices being projected onto the array simultaneously. The resulting spectral image thus contains the spatial and spectral information from each slitlet, and can be reformatted to construct $3.3^{\prime \prime} \times 6.0^{\prime \prime}$ images of the observed object, one image for each resolution element of the grism. The data reduction process yields a 3D data cube which consists of a stack of 2D images spanning the full 1024-pixel wavelength range available from the grism.

The inner knots (NK1 and SK 1) and bow shocks (NB 1/NB 2 and SB 1/SB 2) of HH 212 were observed with the IFU on November 19 and 20, 2002 and November 22 and December 6, 2003, respectively. The 2002 observations were acquired during instrument commissioning; the 2003 data were obtained in service mode. On each occasion, the long (6.0") axis of the IFU was aligned with the jet axis, using a position angle of $22^{\circ} \mathrm{E}$ of $\mathrm{N}$. The HK grism was used, which gives a wavelength range of $1.4-2.5 \mu \mathrm{m}$ and wavelength increment of $1.07 \times$ $10^{-3} \mu \mathrm{m}$. The exposure time per frame was $120 \mathrm{~s}$ with 10 object frames and 10 sky frames taken for each knot and bow shock. Therefore, the total on-source exposure time was $1200 \mathrm{~s}$ for each object.

Halogen flat field and Argon arc exposures were used to calibrate the data; the flats were used to measure the pixel-to-pixel response across the array and to map the extent and location of the spectrum from each slice on the array. The arc exposures were used to wavelength calibrate each spectrum. The bright stars HD 38529 (G4V, $K=4.41$ ) and HIP 29487 (A2V, $K=6.60$ ) were then used to correct for telluric absorption and to flux calibrate the 2002 and 2003 data, respectively.

Data reduction and flux calibration were performed using the orac-DR pipeline software specifically written for the UIST IFU, supplemented by standard STARLINK routines. The data reduction process leading from raw frames to a flux calibrated $3 \mathrm{D}$ data cube is described in detail on the UKIRT IFU webpage ${ }^{1}$. The seeing throughout the observations was $\sim 0.5^{\prime \prime}$.

${ }^{1}$ http://www. jach.hawaii . edu/UKIRT/instruments/uist/ ifu/ifu.html

\section{Results}

Spectra and images were extracted from the data cubes containing the NK 1, SK 1, NB 1/NB 2 and SB 1/SB 2 information (Figs. 2-7). Note that the corresponding Tables referred to below are presented as online materials only.

1. Images were obtained at wavelengths which contained reasonably bright line emission. The instrumental resolution of the $I F U$ in the spectral dimension is 2 pixels corresponding to a wavelength span of $2.14 \times 10^{-3} \mu \mathrm{m}$. Therefore, to get the best signal-to-noise in each line image we extracted images over 4 pixels centred on the line wavelength. Adjacent continuum images were also extracted from each cube; these were used to establish the absence of detectable continuum emission in each region. Consequently, continuum subtraction was not performed.

2. To obtain a representative and reasonably deep spectrum for each feature, data were extracted over the entire spatial extent of the emission-line region in each case. Each spectrum represents the coaddition of data over a spatial area of 2 . $5 \times 3$ ". 5 for the inner knots centered on the knot emission peak. For the inner bows slightly smaller spatial extents (quoted in the figure captions) were used to extract the spectra. The resulting spectra cover a wavelength range from 1.5-2.5 $\mu \mathrm{m}$. The detected $\mathrm{H}_{2}$ emission lines and the [Fe II] line at $1.644 \mu \mathrm{m}$ are labeled in Figs. $2-5$ and the fluxes listed in Tables 1-3 (online material).

We consider below first the inner, bright knots NK 1 and SK 1, and then the more distant, fainter features NB 1/NB 2 and SB $1 / \mathrm{SB} 2$.

\subsection{Inner knots: NK 1 and SK 1}

In the NK 1 and SK 1 spectra (Fig. 2) the continuum flux is close to zero across the entire spectral range and the telluric features have largely disappeared, indicating that the atmospheric absorption correction and flux calibration were relatively accurate. Nevertheless, transmission is poor between $\sim 1.8$ and $\sim 2.0 \mu \mathrm{m}$ and beyond $\sim 2.4 \mu \mathrm{m}$. In these regions, line flux calibrations are therefore still likely to be inaccurate. In order to estimate the error in the flux measurements, the same sky region was used to measure the aperture integrated flux across a broad range of wavelengths. The standard deviation of these values was then used as the average $1 \sigma$ error.

The extracted images of NK 1 and SK 1 are displayed in Figs. 3 and 4. These show a compact, knot-like structure. However, weak emission also extends to the north and south of both knots. This inter-knot emission can be seen in deep VLT $\mathrm{H}_{2}$ 1-0 S(1) images (McCaughrean et al. 2002). The origin of this emission is mysterious. Note that no continuum emission is detected in either knot or stream and no continuum subtraction was performed.

\subsection{Inner Bows: NB 1/NB2 and SB 1/SB2}

The photometric results for the inner bows NB 1/NB 2 and SB 1/SB 2 are presented in Tables 2 and 3. The features NB 1 and NB 2 are distinguishable in our extracted images (Fig. 6) so their flux measurements are listed separately. The features SB 1 and SB 2 are not separated, however, so their flux measurements are presented together. The fluxes of the prominent emission lines in our extracted spectra are considerably weaker than for the inner 

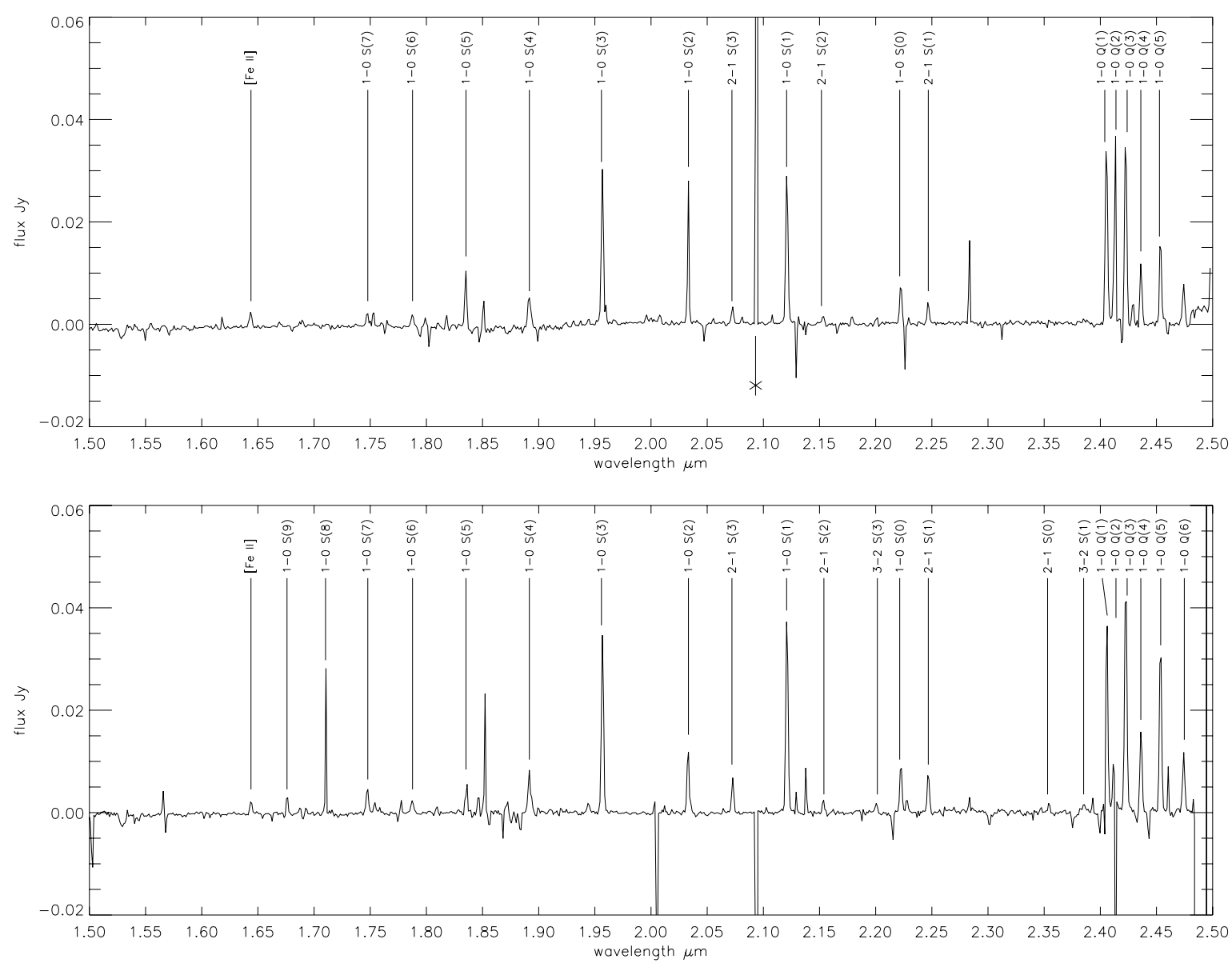

Fig. 2. Spectra of the NK 1 knot (upper) \& the SK 1 knot (lower panel) between $1.5 \mu \mathrm{m}$ and $2.5 \mu \mathrm{m}$. The spectra were extracted over an area of 2 '. $5 \times 33^{\prime \prime} .5$ centered on the emission peak. The asterisk in the top panel indicates a spurious pixel value. Narrow absorption spikes are probably due to bad pixels or cosmic ray hits in the object, sky or standard-star data. Anomolous features may also result from poor telluric correction in parts of the $K$-band window.

knots, the emission being distributed over a larger area. Spectra of the bows are presented in Fig. 5.

The $1-0 \mathrm{~S}(1) S / N$ ratios for NB 1 and SB 1 are $\sim 23$ and $\sim 14$, respectively (determined from the peak flux measurements). Because of the weaker fluxes, images from the higher excitation emission lines were difficult or impossible to extract. In our $\mathrm{H}_{2}$ images (Figs. 6 and 7) the NB 2 bow structure is unresolved whereas NB 1 displays an eastern wing in the 1-0 lines. The SB 1/SB 2 pair displays wing emission typical of bow shocks with the leading bow SB2 responsible for the emission south of the peak.

Note that [Fe II] emission is not detected in either bow. This is not strange since the expected [Fe II] flux level from a bow shock is usually much lower than the level of the 1-0 S(1) flux, as also found specifically for $\mathrm{HH} 212$ by Caratti o Garatti et al. (2006). As with the inner knots, no continuum emission is detected.

\section{Analysis}

\subsection{Excitation}

The molecular excitation of the inner knots are displayed in the Column Density Ratio diagrams of Fig. 8. We first derived the required column of $\mathrm{H}_{2}$ in the upper energy level, $k T_{j}$, of each observed line. However, to permit the relevant error bars to be seen, we then divide the columns by the columns predicted by a uniform medium of temperature $2000 \mathrm{~K}$, taking local thermodynamic equilibrium with an ortho-para ratio of 3 . Hence, a horizontal line on a CDR diagram would indicate a temperature of $2000 \mathrm{~K}$. The effect of added extinction (lower panels) is to provide an improved overall alignment, supporting the interpretation of collisional excitation.

The figures indicate that the measured fluxes for the inner knots and bows of HH 212 are consistent with collisional excitation in shock heated gas. Ortho-para ratios determined from the 1-0 S sequence are close to the thermodynamic equilibrium value of 3 for the inner knots. However, the 1-0 S(0) values for the bows are a factor of 2 below the expected value. This is a consequence of poor sky subtraction near the corresponding wavelength, as evident from the spectra (Fig. 5).

The vibrational excitation is here measured by the integrated flux ratio $R_{v}=(2-1) /(1-0) \mathrm{S}(1)$ and yields the vibrational excitation temperature $5594 \mathrm{~K} /\left(-0.304+\ln \left(R_{v}\right)\right)$ (Eislöffel et al. 2000). The highest excitation is found in the inner knots, $2000 \mathrm{AU}$ from the source where $R=0.10 \pm 0.02$ for NK 1 and $0.16 \pm 0.02$ for SK 1 which correspond to excitation temperatures of $2167 \pm$ $170 \mathrm{~K}$ and $2586 \pm 170 \mathrm{~K}$, respectively.

Lower excitation conditions are found further downwind in each jet, at a distance of $15000 \mathrm{AU}$ from the source where the NB $1 / 2$ and SB $1 / 2$ bow shocks are located. The excitation ratio measured for NB 1 is $0.05 \pm 0.04\left(T_{\mathrm{ex}}=1698 \pm 326 \mathrm{~K}\right)$. For SB 1 a ratio of $0.06 \pm 0.03\left(T_{\mathrm{ex}}=1807 \pm 277 \mathrm{~K}\right)$ is found. These results are not in complete agreement with the excitation 

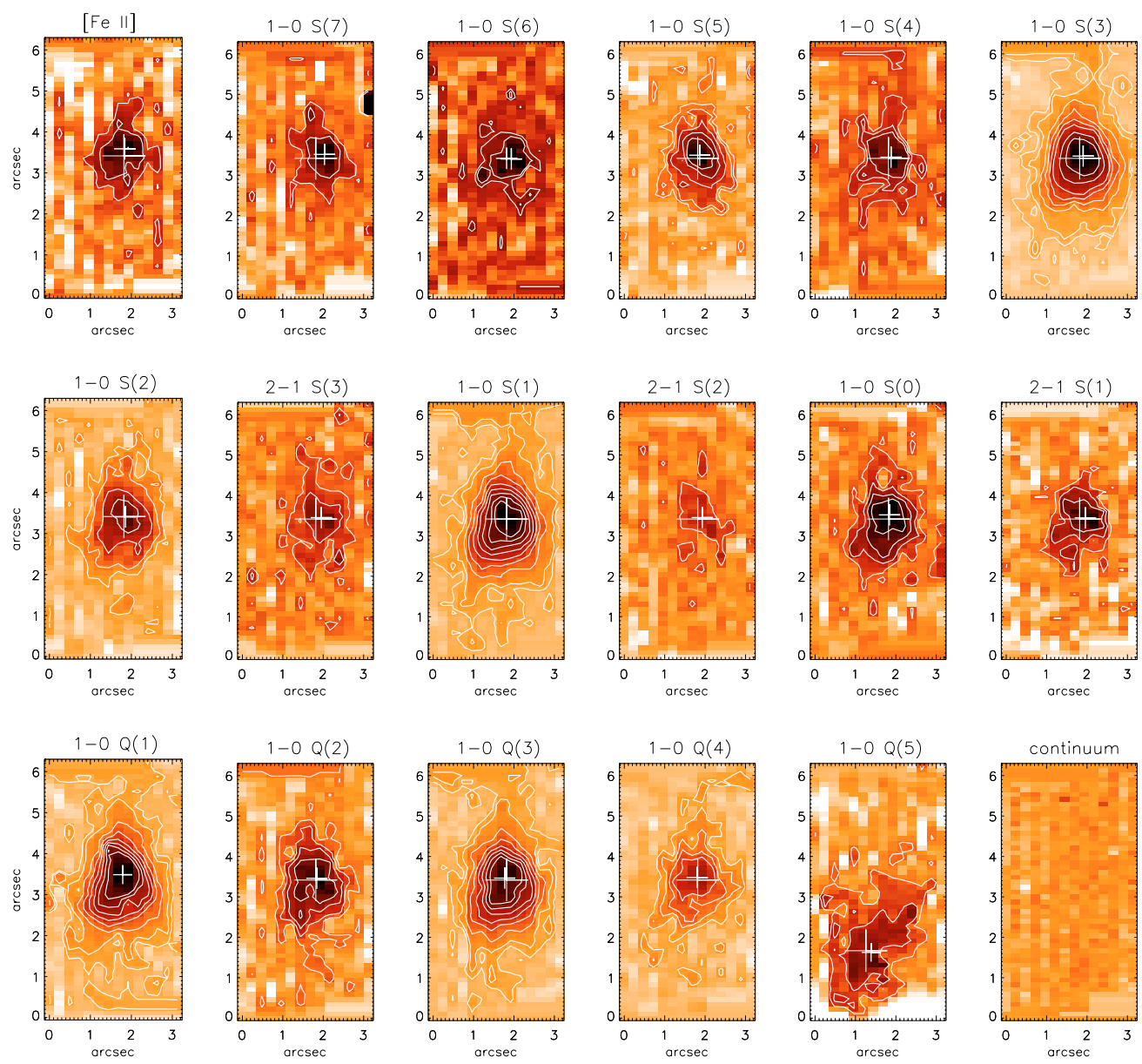

Fig. 3. Extracted line emission images for NK 1. The peak flux positions were estimated by Gaussian fitting of the image peaks and are indicated by the crosses. $X$-axis offsets increase toward the west; $Y$-axis offsets increase roughly northward along the jet axis (see Fig. 1). A continuum emission image, from data between $1.7 \mu \mathrm{m}$ and $1.71 \mu \mathrm{m}$, is also provided. The large faint cross in each panel indicates the peak of the $1-0 \mathrm{~S}(1)$ line.

temperatures derived from deep $K$-band spectroscopy at the NTT by Tedds et al. (2002). They found high excitation temperatures for both the inner knots as well as the bow shocks ( $T_{\mathrm{ex}} \simeq$ $2300 \mathrm{~K})$. However, when we consider only the peak flux in each case, i.e. by extracting spectra just from the emission peak in our data cubes, rather than from the entire knot, equally high excitation temperatures are found (although the accompanying errors are relatively large) for the inner knots and bows. This suggests that the IFU observations are more sensitive to the lower excitation extended emission which extends away from the bows. Note that the spectra presented by Tedds et al. (2002) were obtained with a long-slit, which did not pass through the lateral wings of each knot/bow shock.

For SK 1, the $\log (\mathrm{CDR})$ is not linearly related to $T_{j}$ due to the high 3-2 S(3) column. Therefore, the excited gas is not of constant temperature. A curved surface of varying temperature is consistent with this result, i.e. as one would expect from excitation in a bow shock, rather than a planar shock. SK 1 does indeed have a "triangular", bow-shaped morphology. For NK 1, however, a linear fit is clearly possible in Fig. 8. Deeper observations of emission lines originating from the higher vibrational levels are required to better constrain the models.

\subsection{Extinction}

The $K$-band extinction for each knot and bow is determined using the ratio $R_{K}=(1-0 \mathrm{Q}(3)) /(1-0 \mathrm{~S}(1))$ as described in
O'Connell et al. (2005). The differential extinction between the two lines is then given by $2.5 \log \left(R_{K}\right)+0.385$. From this, we estimate $A_{K}$ values of $1.2 \pm 0.2 \mathrm{mag}$ and $1.4 \pm 0.2 \mathrm{mag}$ for NK 1 and SK 1. Much lower extinctions of $A_{K}<0.5 \mathrm{mag}$ are found for NB 1 and SB 1, supporting the idea that they have broken out of the dense protostellar envelope and are now propagating through a medium of reduced density.

CDR diagrams for NB 1, NB 2 and SB 1/SB 2 have also been obtained but are not presented. The lack of detections of high excitation transitions makes it impossible to identify a temperature dependence. The CDRs of all the Q-branch lines relative to the 1-0 S-Branch lines constrain the extinction. An $A_{K}$ extinction close to zero produces the best results, confirming that the foreground column density is low.

The low excitation is consistent with the lack of detectable optical emission from the outer bows. This requires these bows to be weak shocks in which the molecules remain intact even at the apex of the bow, as would appear to be the case from the images (Zinnecker et al. 1998; McCaughrean et al. 2002).

\subsection{Peak flux positions}

An advantage of the IFU is that multi-wavelength information is gathered simultaneously. Positional variations in the molecular excitation can therefore be accurately determined without the misalignment uncertainty produced by image co-registration. The left panels of Fig. 9 display the NK 1 and SK 1 1-0 S(1) 

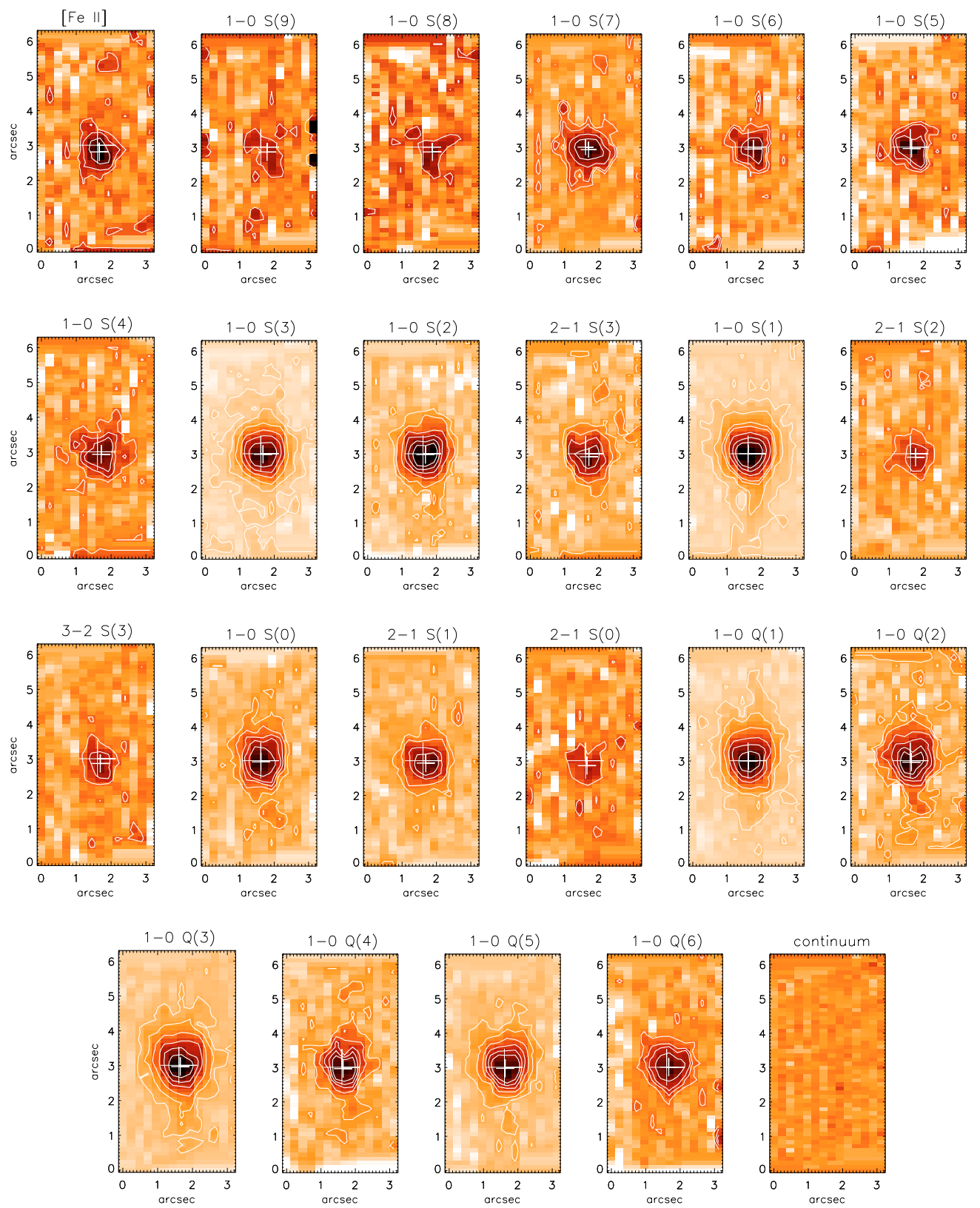

Fig. 4. Extracted line emission images for SK 1. See Fig. 3 for details.

images overlaid with [Fe II] flux contours. Whereas the shift in the peak position is clearly detected between the 1-0 S(1) and [Fe II], these images show that the rest of the emission is also shifted; the bulk of the [Fe II] emission lies ahead of the 1-0 S(1) emission (in the jet flow direction), as would be expected if they were forward moving bow shocks with highexcitation tips.

Also shown are maps of the $(2-1) /(1-0) \mathrm{S}(1)$ ratio with overplotted contours of the 1-0 S(1) (middle panels) and [Fe II] (right panels) emission. For NK 1, no variation can be detected in the excitation map but the SK 1 ratio map shows a clear increase in the vibrational excitation towards the south with the [Fe II] shifted in the same direction. Therefore, SK 1 closely corresponds to the general structure expected from a bow shock with excitation increasing towards the bow apex.

The peak flux positions are given as offsets relative to the peak of the $\mathrm{H}_{2}$ 1- $0 \mathrm{~S}(1)$ line in Table 1 . These were determined by fitting a $2 \mathrm{D}$ Gaussian using a STARLINK object detection routine. This method thus determines the peak under the condition that the intrinsic spatial profiles are Gaussian. The detection threshold was set in order to include only the higher value pixels (typically about $15-25$ ) which are near the peak position. The pixel scale is $0.24^{\prime \prime}$ in the $x$-direction and $0.12^{\prime \prime}$ in the $y$-direction. However, the errors in the peak locations are considerably less than the pixel size and are related to the seeing. It is straightforward to show that the theoretical error on the peak position through the Gaussian fitting is approximately given by $\sigma /(S / N)$ where $\sigma=F W H M / 2.35$ is the standard deviation of the Gaussian and $S / N$, the signal to noise ratio, is the ratio of peak flux to the noise level for each line transition. Therefore, the high $S / N$ ratios for the 1-0 S(1) lines of NK 1 and SK 1 of $\sim 49$ and $\sim 110$, respectively, lead to small resultant errors, as indicated in Figs. 10 and 11.

The absolute positional errors are significantly larger, being related to the telescope pointing. However, the integral field technique may yield much smaller relative errors through the 

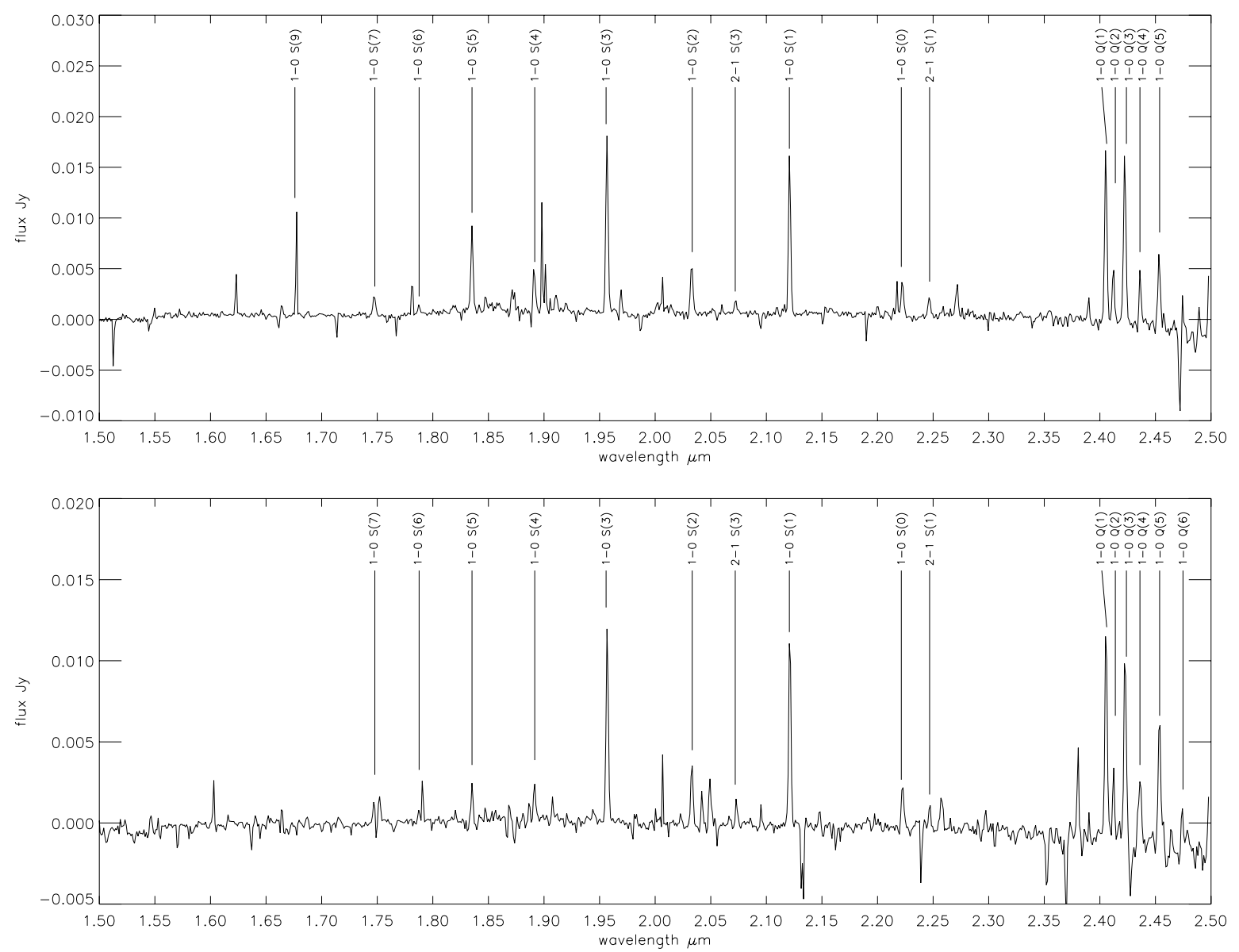

Fig. 5. Spectra of the NB 1 bow (top panel) and SB 1 and SB 2 (lower panel) between 1.4 and $2.5 \mu \mathrm{m}$. The NB 1 spectrum is extracted over an area of $1^{\prime \prime} .8 \times 1^{\prime \prime} .8$ centered on the NB 1 peak. The SB 1 and SB 2 spectrum is extracted over an area of $2{ }^{\prime \prime} .0 \times 22^{\prime \prime} 0$ centered on the SB 1 peak.

above method. Moreover, a limit to the accuracy can be estimated from the data by comparing the peak locations from lines originating from the same upper energy level yet well separated in wavelength. On physical principles, the line pairs possess identical flux distributions, differing only in relative flux, possibly varying if there are sharp spatial gradients in extinction. Most significantly, for SK1, the peak locations from each of the five pairs agree to within $\sim 0.02^{\prime \prime}$ except for the $y$-offsets from the 1-0 S(4) and $\mathrm{Q}(6)$ transitions where the agreement is still within $2 \sigma$ (see Fig. 11).

For NK1, there are more significant discrepancies evident in the peak locations of some of the weaker para $1-0 \mathrm{H}_{2}$ line pairs (Fig. 10, the difference reaching $\sim 0.1^{\prime \prime}$ in the $y$-offset). Given the accuracy of the SK 1 line peaks, this suggests that the NK 1 discrepancies root from an intrinsically non-Gaussian (asymmetric) central region to the line profile. Then, a convolution with the seeing and signal-to-noise would tend to remove the skewness, shifting the peak position. This is indeed supported by the line images of NK 1 (Fig. 3) which display a steeper fall-off in flux towards the north-west.

The peak position offsets are plotted against the upper level temperature of each transition in Figs. 10 and 11 for NK 1 and SK 1, respectively. Again, note that we assume Gaussian fits. The error is then related to the seeing of $0.5^{\prime \prime}$ and the signal-to-noise.

For NK 1 no trend can be seen along the outflow axis (in the $y$-direction). However, a clear trend is found perpendicular to the flow axis (in the $x$-direction). This unexpected result for an advancing bow shock indicates that the excitation increases slightly from east to west (left to right in all the figures presented here).

For SK 1 the higher excitation lines are shifted in the $y$-direction towards the south, away from the central source. Interestingly, in the $x$-direction the same trend is measured as in NK 1, with increasing excitation from east to west.

The offsets measured for the bows NB and SB show no trends (not displayed here). The detected emission is too weak to locate the higher excitation line emission peaks. More sensitive observations are required to complete such an analysis.

\section{Models}

\subsection{Interpretations}

We wish to explain the spatial variation of the gas excitation evident as a) the offset of the [Fe II] emission ahead of the $\mathrm{H}_{2}$ peaks in the inner knots NK1 and SK1, b) a shift in the higher excitation $\mathrm{H}_{2}$ emission of SK1 towards the south and c) westward shifts in the higher excitation $\mathrm{H}_{2}$ emission lines across both inner knots. The variation corresponds to a change in gas temperature of order of $1000 \mathrm{~K}$ across a distance of $0.1^{\prime \prime}$ or $\sim 7 \times 10^{14} \mathrm{~cm}$. We consider three possibilities below.

The cooling length across a radiative shock, in which the shock front and cooling layer are distinct, is very short, typically under $\sim 10^{13} \mathrm{~cm}$. Alternatively, with ambipolar diffusion, the length scale of a C-type shock is $\sim 10^{15} /\left(n_{i} \mathrm{~cm}^{-3}\right) \mathrm{cm}$ where $n_{i}$ is the ion number density (see Smith \& Brand 1990a). Note, 

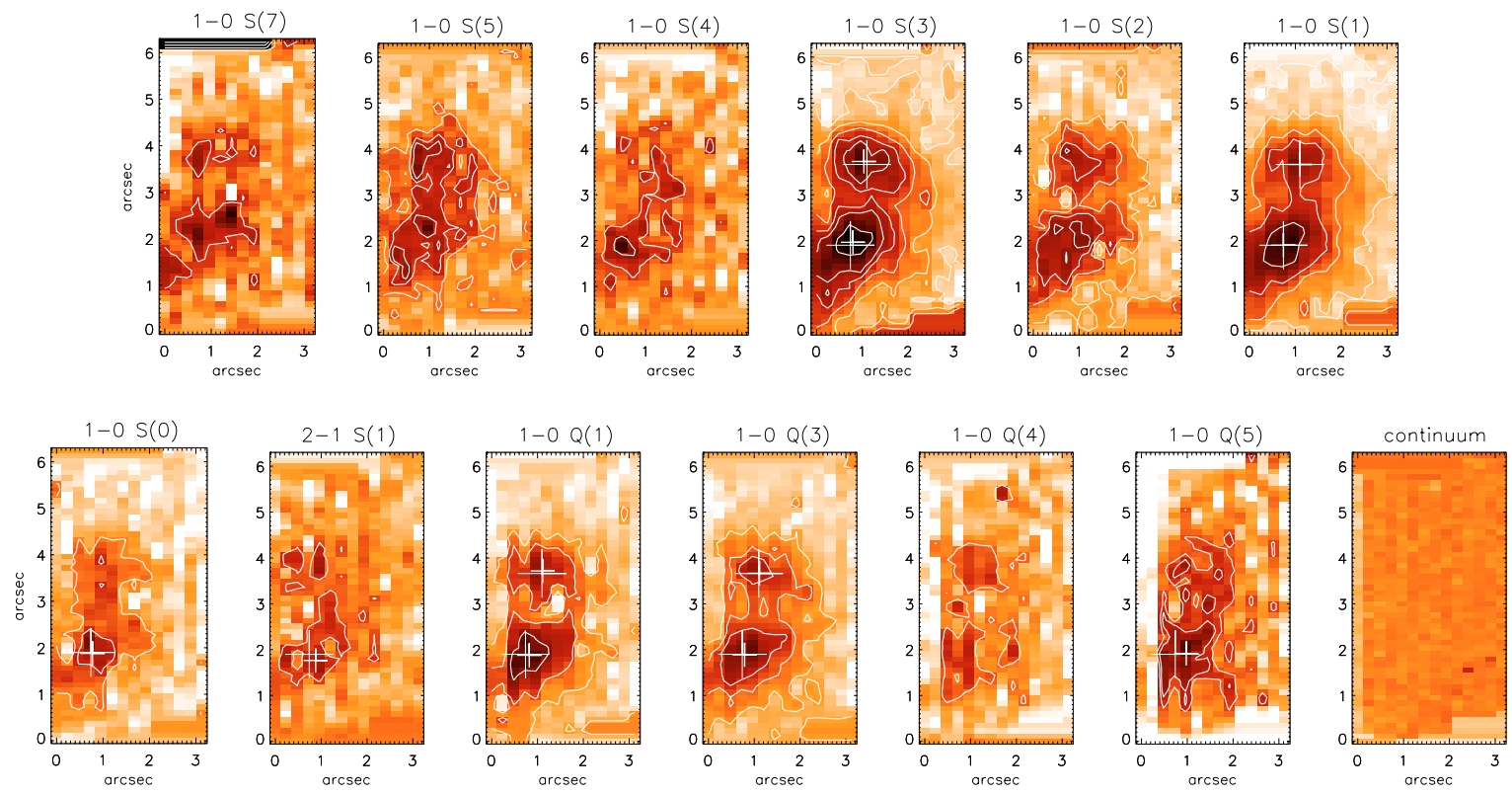

Fig. 6. Extracted line emission images of NB 1 and NB 2. NB 2 is the northern, upper-most feature. Where possible, the positions of the peaks were determined via Gaussian fitting and are indicated by crosses (see Fig. 3 for further details). A continuum emission image, from data between $1.7 \mu \mathrm{m}$ and $1.71 \mu \mathrm{m}$, is also provided. The large faint cross in each panel indicates the peak of the $1-0 \mathrm{~S}(1)$ line.
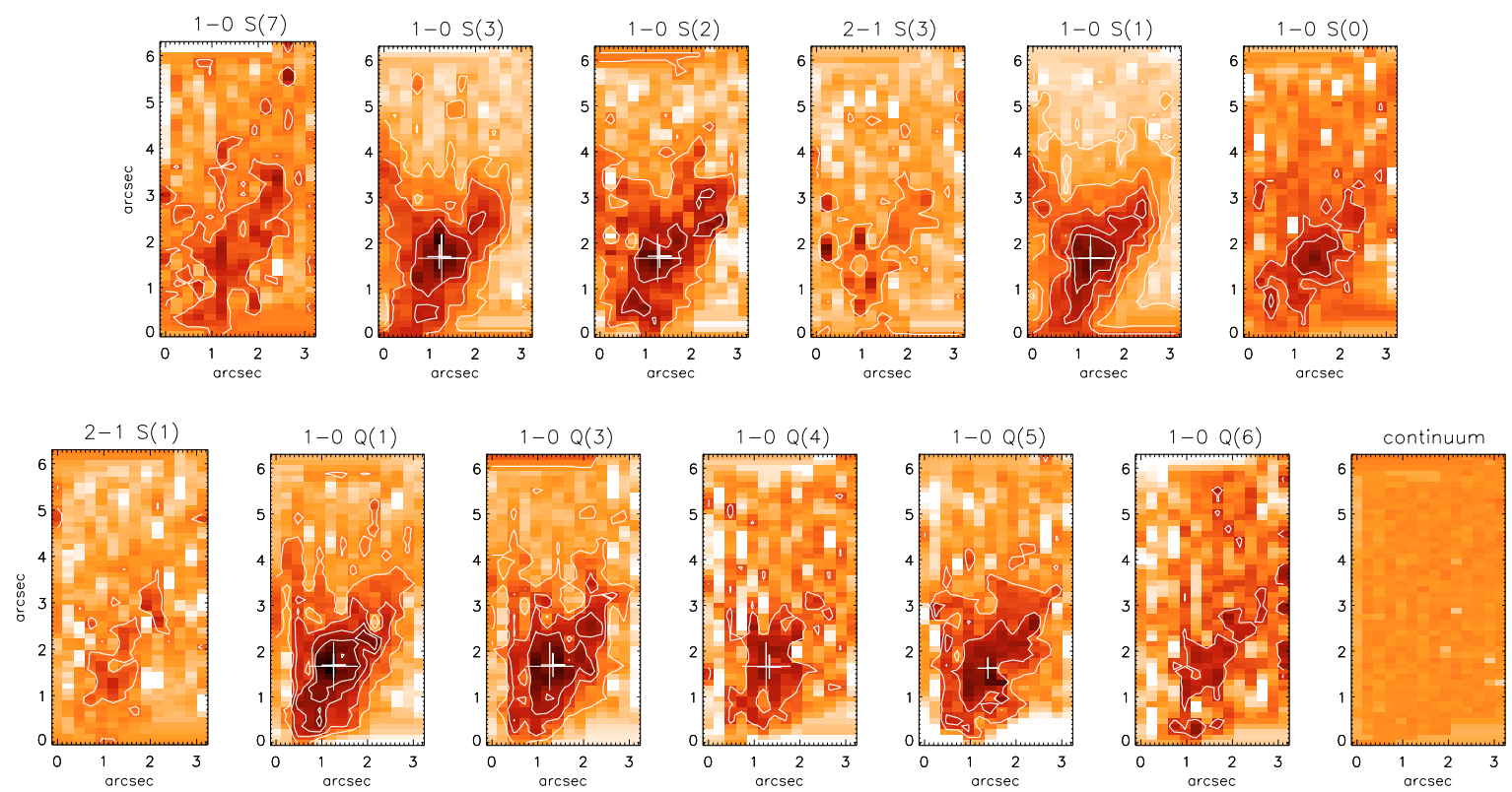

Fig. 7. Extracted line emission images of SB 1/SB 2 (see Fig. 3 for details). These two faint knots, labelled in Fig. 1, are essentially unresolved here. The large faint cross in each panel indicates the peak of the 1-0 S(1) line.

however, that the section of the shock across which the gas remains vibrationally excited is a moderate fraction of this since the gas is no longer excited once accelerated (Smith \& Brand 1990b). Nevertheless, provided $n_{i}$ is less than $\sim 1 \mathrm{~cm}^{-3}$, the shock thickness could contribute to the excitation gradient.

A second possibility is that the excitation varies smoothly along the surface of a curved bow shock. Since the shocks are clearly curved into bow-like shapes, this almost certainly is important. Modelling which includes the above two effects is carried out below.

Thirdly, two adjacent shocks of different strength may contribute. The shocks could be associated with forward and reverse shocks, the latter possibly taking the form of a Mach disk. Given the smooth variations across the inner knots, the two cooling zones would have to be only partially resolved. Although this cannot be excluded, the presence of emission from the iron ion in the inner knots, which is also displaced towards the location of high molecular excitation, suggests that the temperature variations occur across the surface of a bow shock.

Possible reasons for the displacement of higher excitation emission to the west in both knots are as follows.

(1) The driving source is moving towards the west relative to the surrounding cloud. A pair of north-south pulsating jets, symmetric relative to the protostar, are subject to increased ram pressure on their west boundary, resulting in a slightly higher excitation. In this case the misalignment of the jet and 

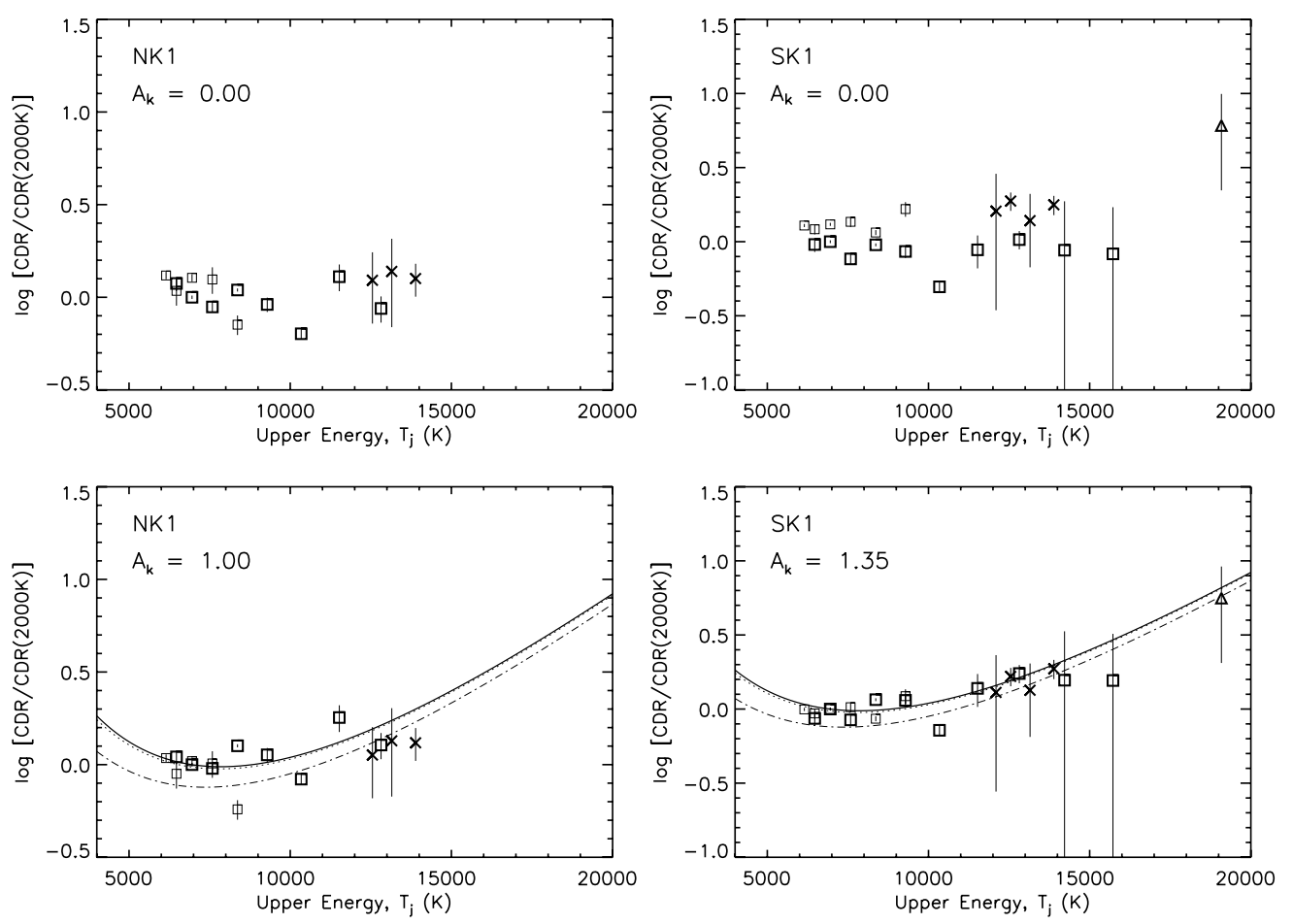

Fig. 8. Column density ratio (CDR) diagrams for NK 1 (left panels) and SK 1 (right panels). The upper panels display data assuming no extinction whereas in the lower panels the extinction has been adjusted to minimise the difference between the 1-0 S-branch and Q-branch lines originating from the same upper energy level. $\mathrm{H}_{2} v=1-0$ transitions are represented by squares, $2-1$ transitions by crosses and 3-2 by triangles. The faint squares represent the 1-0 Q-branch measurements. The CDRs predicted by the best fit J-type bow shock model are also shown; the solid line represents the first vibrational level, dotted represents the second, and the dot-dashed line shows the third vibrational level.
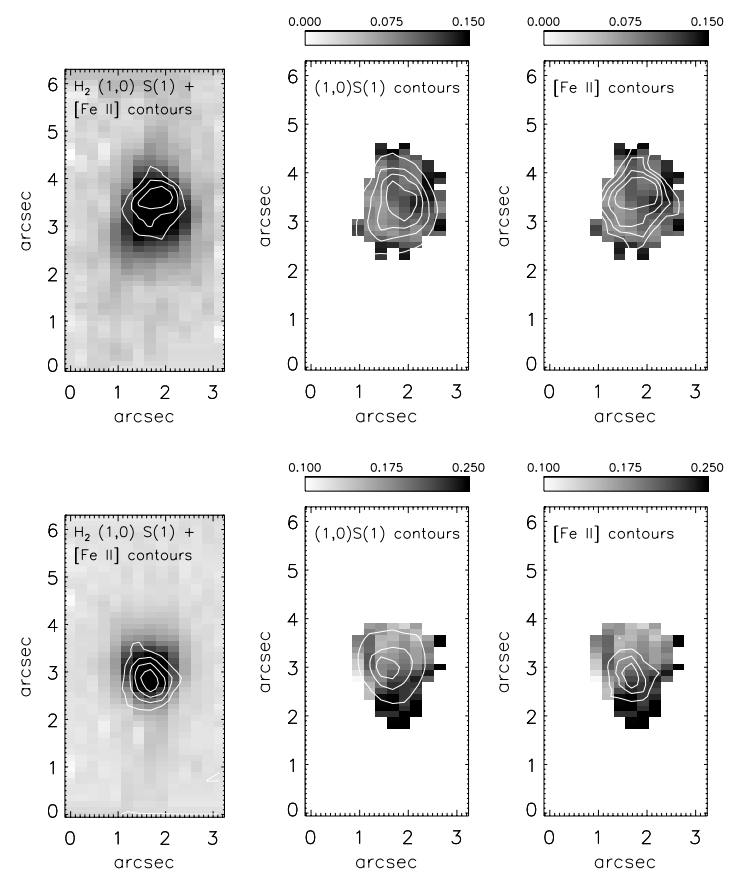

Fig. 9. Left: $\mathrm{H}_{2} 1-0 \mathrm{~S}(1)$ image of $\mathrm{NK} 1$ (upper panels) and SK 1 (lower panels) overlaid with [Fe II] contours. Middle and right: $\mathrm{H}_{2}(2-1) /(1-0) \mathrm{S}(1)$ ratio image of NK 1 (upper) and SK 1 (lower) overlaid with $\mathrm{H}_{2} 1-0 \mathrm{~S}(1)$ and $[\mathrm{Fe} \mathrm{II}]$ contours.

counter jet would not be caused by the momentum of the streaming cloud because the jets are misaligned in the opposite sense, i.e. with the jets inclined towards the approaching cloud material.
(2) The outflow, possibly with a small angle of precession, has burrowed a tunnel through high density clumpy circumstellar gas. Higher excitation would be expected where the jets presently happen to abrade denser regions along the tunnel edges.

(3) If the magnetic field is oblique to the jet axis, the asymmetry would result in preferential molecular excitation toward one flank of the bow. In this case the magnetic field lines would have to be orientated in different directions for each bow. Below, a bow shock model is used to test this scenario.

(4) Jet shear could result in a systematic variation in shock strength across the jet. As shown by Davis et al. (2000), there is a variation in the radial velocity across the knots of order of a few $\mathrm{km} \mathrm{s}^{-1}$. If this is due to shear rather than rotation, then the velocity difference must be quite large. With a jet flow at $5^{\circ}$ to the plane of the sky, the observed radial velocity differences require a shear along the jet axis of $10-30 \mathrm{~km} \mathrm{~s}^{-1}$. This would significantly displace the point of impact of the bow shocks transverse to the jet axis.

\subsection{A bow shock model for the inner knots}

We attempt to model the inner knots NK1 and SK1 using various bow shock configurations. We employ a steady state model which assumes a fixed geometry. The three dimensional curved bow surface is described by

$$
Z / L_{\text {bow }}=(1 / s)\left(R / L_{\text {bow }}\right)^{s},
$$

where $Z$ and $R$ are cylindrical coordinates. The size of the bow is determined through the choice of $L_{\text {bow }}$ and the shape or curvature is fixed through the parameter $s$. In order to constrain the model to match the observed set of data, several parameters are 

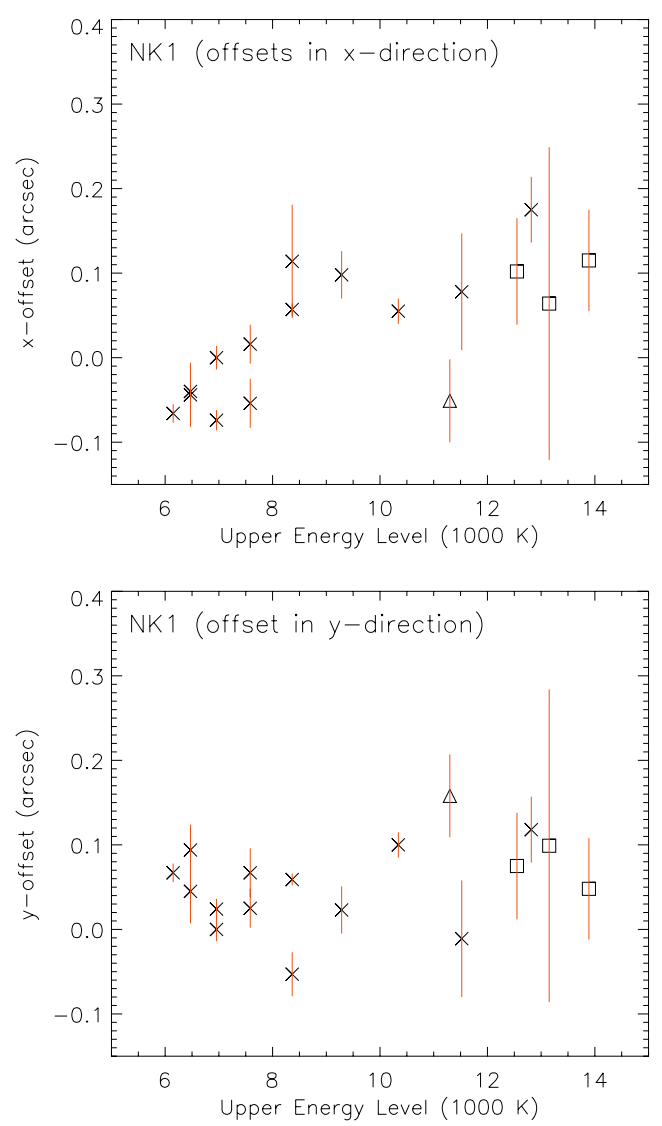

Fig. 10. The $x$ (upper panel) and $y$ (lower panel) peak flux positions relative to the 1-0 S(1) line are plotted against the upper energy level of each transition for NK 1 . Crosses represent the $v=1-0$ transitions, squares the $v=2-1$, and the triangle represents the $[\mathrm{Fe} I \mathrm{II}]{ }^{4} \mathrm{D}_{7 / 2}-$ ${ }^{4} \mathrm{~F}_{9 / 2}$ transition at $1.644 \mu \mathrm{m}$. The estimated systematic errors are indicated by error bars. Note that, in the top panel, the lower-excitation lines appear at negative (eastern) offsets, while the higher-excitation $\mathrm{H}_{2}$ and [Fe II] lines appear at positive (western) offsets. No such trend is seen along the $y$-axis (the flow axis) in the lower panel.

varied including the bow velocity, pre-shock density, magnetic field strength, ion fraction as well as geometric considerations such as the orientation with respect to the line of sight. The same model was successfully employed to interpret the bow shocks in $\mathrm{HH} 240$ and $\mathrm{HH} 211$. The method of parameter exploration and the accuracies and uncertainties involved are described in detail in O'Connell et al. (2004) and O'Connell et al. (2005). The means of calculating the [Fe II] emission and similar image analysis were presented by Khanzadyan et al. (2004).

NK 1 and SK 1 are similar in terms of their luminosities and measured flux ratios. A consistent model is found to be a J-type bow shock for which sufficient ions are assumed so that the magnetic field is frozen to the neutral gas. Molecular hydrogen dissociates when the shock speed exceeds $\sim 24 \mathrm{~km} \mathrm{~s}^{-1}$. The model parameters are listed in Table 4 . The model 1-0 S(1) luminosity and predicted line ratios are given in Table 5 alongside the measured values for NK 1 and SK 1 .

We also present the model images for SK 1 in Fig. 13 since this knot has a slightly higher flux and signal-to-noise ratio $(S / N=110)$. The derived shape of the model bow, with extended molecular flanks, is necessary to explain the low excitation (which is generally very high for planar J-shocks), as shown by the fits in Fig. 8. On the other hand, the quite low bow speed of $36 \mathrm{~km} \mathrm{~s}^{-1}$ (relative to the upstream jet flow) maintains the
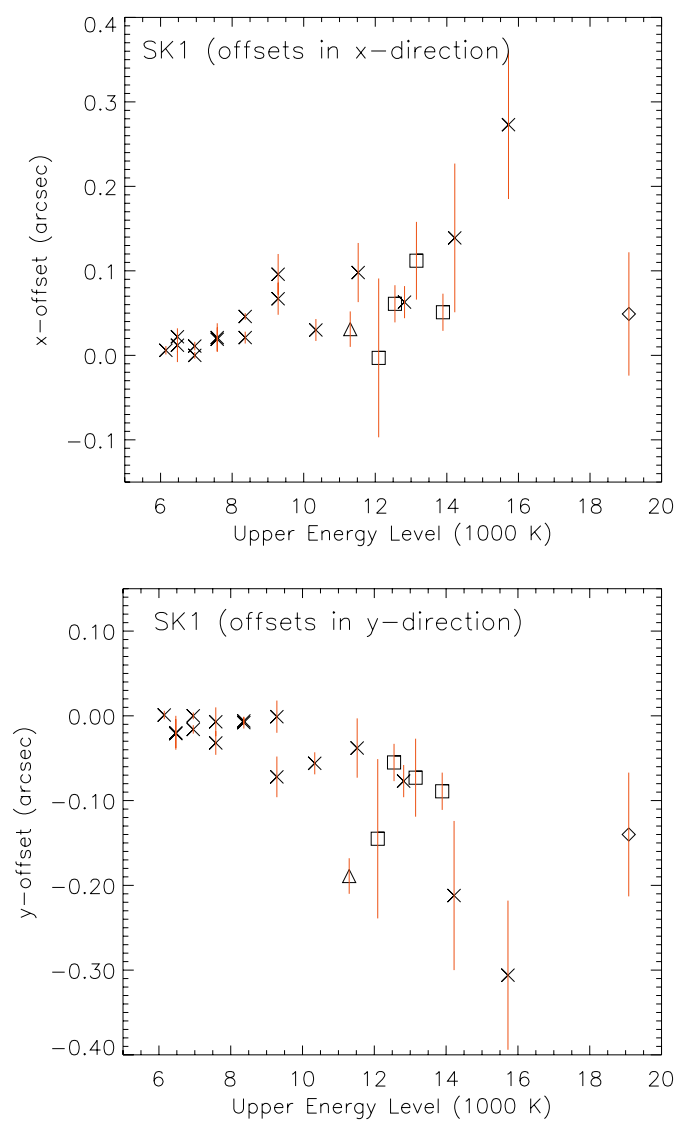

Fig. 11. The $x$ (upper panel) and $y$ (lower panel) peak flux positions relative to the 1-0 S(1) line are plotted against the upper energy of each transition for SK 1. The symbols are as in Fig. 10 and the diamond represents the 3-2 S(3) peak position. In SK 1, the excitation increases from east to west (upper panel - like NK 1) but also from north to south (lower panel) along the jet axis. Peak postions were derived from the convolved data.

Table 4. Model parameters derived to fit the inner knots NK 1 and SK 1 with a J-type shock. $n=n(\mathrm{H})+2 n\left(\mathrm{H}_{2}\right)$ is taken as the hydrogen nucleon density.

\begin{tabular}{ll}
\hline \hline Parameter & NK 1/SK 1 \\
\hline Size, $L_{\text {bow }}$ & $3.9 \times 10^{15} \mathrm{~cm}$ \\
(1) H density, $n$ & $9.0 \times 10^{4} \mathrm{~cm}^{-3}$ \\
(2) Molecular fraction $f^{*}$ & 0.2 \\
(3) Alfvén speed, $v_{\mathrm{A}}$ & $8 \mathrm{~km} \mathrm{~s}^{-1}$ \\
Magnetic field & $1.3 \mathrm{mG}^{-1}$ \\
(4) Bow velocity, $v_{\text {bow }}$ & $36 \mathrm{~km} \mathrm{~s}^{-1}$ \\
(5) Angle to l.o.s. & $90^{\circ}$ \\
(6) Shape parameter s & 1.5 \\
(7) Field angle, $\mu$ & $-54^{\circ}$ \\
\hline
\end{tabular}

* The molecular fraction is given by $f=n\left(\mathrm{H}_{2}\right) / n$ so when $f=0.5$ the gas is fully molecular.

compact shape of the features (whereas fast J-shocks generate long tailed emission line regions).

The alternative C-type model generally consists of an apex region which is J-type and bow flanks which are curved $\mathrm{C}$-shocks in which the molecules are not dissociated. We cannot exclude this model with the present data. However, we find that a paraboloidal bow of speed $\sim 65 \mathrm{~km} \mathrm{~s}^{-1}$ emits excessive $[\mathrm{Fe}$ II] emission from the bow cap. A more acute bow with shape 

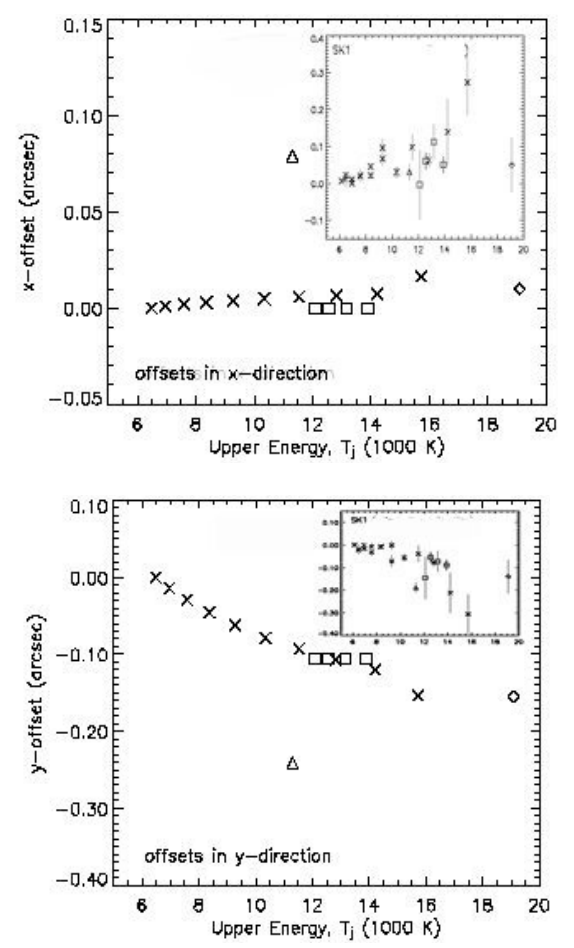

Fig. 12. The $x$ (upper panel) and $y$ (lower panel) offsets relative to $1-0 \mathrm{~S}(1)$ position predicted by the model. Note that $6.7 \times 10^{15} \mathrm{~cm}$ corresponds to $1^{\prime \prime}$ at the adopted distance. The symbols are as defined in Fig. 10. To predict the offsets a Gaussian fit was performed to locate the peak position. In order not to include faint emission, the Gaussian fit was limited to values above $40 \%$ of the maximum value (as was the case in determining the peaks for the observed images). These plots are here compared in the inset panels to the observational data in Fig. 11 for SK1.

$s=1.5$, as also advanced for S233 N6 (Khanzadyan et al. 2004), is consistent with the HH 212 knot fluxes.

In order to introduce asymmetry into the modelled structure the magnetic field was orientated at an angle of $-54^{\circ}$ relative to the direction of motion of the bow (the geometry is described in O'Connell et al. (2004)). Note that a high Alfvén speed is necessary for the magnetic field to provide the asymmetry in a J-type bow (Table 4).

In order to match the seeing, the model images were convolved with a Gaussian of $F W H M=3.5 \times 10^{15} \mathrm{~cm}$ which is equivalent to the seeing throughout the observations of $\sim 0.5^{\prime \prime}$ at the adopted distance of $460 \mathrm{pc}$. The greatest similarity to the observed bow appearance and line ratios was found for a bow of velocity $36 \mathrm{~km} \mathrm{~s}^{-1}$ and pre-shock hydrogen nucleic density of $9.0 \times 10^{4} \mathrm{~cm}^{-3}$. The resulting bow is displayed in Fig. 13, which shows the predicted appearance in the 1-0 S(1) and 2-1 S(1) lines of $\mathrm{H}_{2}$ as well as the [Fe II] emission which is generated from near the bow apex. Both the unconvolved and convolved structures are shown. Figure 13 also shows the positions of the predicted emission peaks. The peaks in the model data were determined using the same method as was used to find the observed peak positions described above.

In Fig. 12, the predicted $\mathrm{x}$ and $\mathrm{y}$ peak positions from the SK 1 model bow relative to the 1-0 S(1) peak are plotted against the upper energy level for a number of emission line images (most of which were detected for SK 1). The trend in the $y$-direction bears a close resemblance to the observed offset positions for SK 1 in Fig. 11. Higher excitation line peaks are located closer to the front of the bow where the strongest
Table 5. Observed and predicted bow shock luminosities and line ratios.

\begin{tabular}{lccc}
\hline \hline Line & Observed $^{*}$ & Dereddened $^{\dagger}$ & $\begin{array}{c}\text { J-type } \\
\text { Model }\end{array}$ \\
\hline $\mathrm{NK} 1$ & & & \\
\hline $\mathrm{H}_{2}(1,0) \mathrm{S}(1)$ & $8.0 \times 10^{-4}$ & $2.0 \times 10^{-3}$ & $2.0 \times 10^{-3}$ \\
$\mathrm{H}_{2}(2,1) \mathrm{S}(1)$ & $8.1 \times 10^{-5}$ & $2.0 \times 10^{-4}$ & $2.3 \times 10^{-4}$ \\
$\mathrm{H}_{2}(3,2) \mathrm{S}(3)$ & - & - & $6.0 \times 10^{-5}$ \\
{$[\mathrm{Fe} \mathrm{II}]{ }^{4} \mathrm{D}_{7 / 2}-{ }^{4} \mathrm{~F}_{9 / 2}$} & $1.2 \times 10^{-4}$ & $5.3 \times 10^{-4}$ & $3.1 \times 10^{-4}$ \\
$(2,1) /(1,0)$ ratio & $0.10(0.02)$ & $0.10(0.02)$ & 0.11 \\
{$[\mathrm{Fe} \mathrm{II}] /(1,0)$ ratio } & $0.15(0.02)$ & $0.27(0.04)$ & 0.15 \\
\hline $\mathrm{SK} 1$ & & & \\
\hline $\mathrm{H}_{2}(1,0) \mathrm{S}(1)$ & $9.8 \times 10^{-4}$ & $3.4 \times 10^{-3}$ & $2.0 \times 10^{-3}$ \\
$\mathrm{H}_{2}(2,1) \mathrm{S}(1)$ & $1.5 \times 10^{-4}$ & $5.2 \times 10^{-4}$ & $2.3 \times 10^{-4}$ \\
$\mathrm{H}_{2}(3,2) \mathrm{S}(3)$ & $3.4 \times 10^{-5}$ & $8.4 \times 10^{-5}$ & $6.0 \times 10^{-5}$ \\
{$[\mathrm{Fe} \mathrm{II}]{ }^{4} \mathrm{D}_{7 / 2}-{ }^{4} \mathrm{~F}_{9 / 2}$} & $6.6 \times 10^{-5}$ & $4.5 \times 10^{-4}$ & $3.1 \times 10^{-4}$ \\
$(2,1) /(1,0)$ ratio & $0.16(0.02)$ & $0.16(0.02)$ & 0.11 \\
{$[\mathrm{Fe} \mathrm{III}] /(1,0)$ ratio } & $0.07(0.02)$ & $0.13(0.04)$ & 0.15 \\
\hline
\end{tabular}

* The $3 \sigma$ detection limits are $5.1 \times 10^{-5} L_{\odot}$ and $6.4 \times 10^{-5}$ for NK 1 and SK 1 respectively. Luminosities are expressed in units of $L_{\odot}$ and a distance of $460 \mathrm{pc}$ is adopted.

$\dagger$ Luminosities have been dereddened using $K$-band extinctions of 1.0 and $1.4 \mathrm{mag}$ for NK 1 and SK $1 . H$-band extinctions of 1.6 and $2.1 \mathrm{mag}$ were used to deredden the [Fe II] luminosities (using $A_{\mathrm{H}}=1.56 \times A_{K}$ from Rieke \& Lebofsky (1985)).

excitation conditions are experienced. The introduction of an oblique magnetic field direction induces an asymmetry in the bow appearance. However, the offset trend in the $x$-direction does not match the observed trend. Firstly, the trend takes place on a much smaller scale than the observed shifts in positions and, secondly, the [Fe II] peak position is offset by a significant amount relative to the $\mathrm{H}_{2}$ lines, unlike the observed offset.

Figure 14 shows an image of the model 2-1 S(1)/1-0 S(1) vibrational excitation ratio. The excitation is seen to steadily increase towards the bow front. For comparison with Fig. 9 the predicted [Fe II]] emission is indicated by the overplotted contours. The similarities between the observed appearance and luminosities of the knots with the predictions of the best-fit model lead us to conclude that the bright structures aligning the inner jet regions of $\mathrm{HH} 212$ are consistent with bow shocks. However, the asymmetry is probably due to some other effect rather than an oblique magnetic field (e.g. rotation or density gradient). Higher resolution observations should reveal the underlying curved structures which trace the outflowing jet material.

\subsection{Double bow shocks}

The appearance of the double bow shocks NB 1/NB 2 and SB 1/SB 2 is puzzling. Close examination of the jet knots in the high resolution image presented in McCaughrean et al. (2002) reveals that some of the knots can also be resolved into two components.

Variations in jet velocity can steepen and give rise to a series of double shocks which are occasionally visible as double bows in synthetic images of the 1-0 S(1) emission line derived from numerical simulations (Völker et al. 1999). Double shocks may also be due to the presence of a forward ambient shock front and a reverse Mach disk. In both cases, the separation between the two shock components is reduced by the strong cooling of the shocked gas. The clear separation seen in the outer bows would then be due to the lower density medium in these regions. 

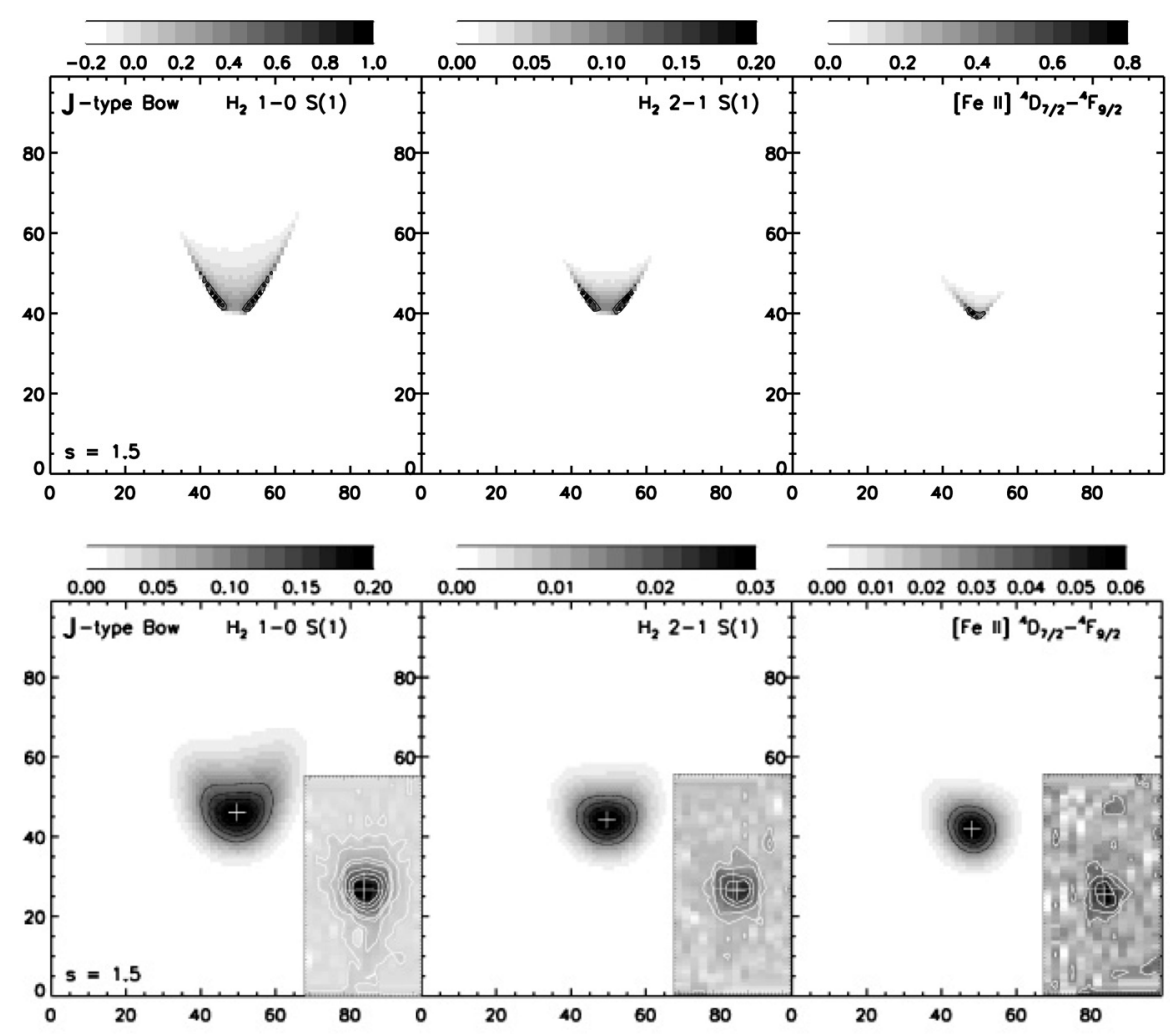

Fig. 13. A J-type bow shock model for SK 1 . The $x$ and $y$ axis units represent $3.9 \times 10^{14} \mathrm{~cm}$, equivalent to $0.057^{\prime \prime}$ at the adopted distance of 460 pc. In the lower panel the images have been smoothed with a Gaussian of $F W H M=9$ pixels, which is equivalent to $3.5 \times 10^{15} \mathrm{~cm}$ and $\sim 0.5^{\prime \prime}$ (the average seeing throughout the observations). The crosses mark the positions of the peak emission. The equivalent observed images for SK1 are also displayed.

Such a configuration also provides an explanation for the inter-knot separation as illustrated in Fig. 15. Whereas the forward shock results in the entrainment of ambient material which is deflected outward, a curved reverse shock has the effect of entraining and refocusing the jet material into a more collimated flow. At high densities the forward and reverse shocks come into close contact and the reverse shock is forced to adopt a curved shape to match the forward bow. The reverse shock focuses and propels the jet material into a thin stream which approaches the forward shock. The high momentum jet penetrates through the forward shock at or close to the apex forming a continuous stream of inter-knot emission due to shock heating where the shearing of flow velocities occurs. This effect is also observed in numerical simulations, leading to a thin nose of material in advance of the bow shock which then appears as two shoulders (Rosen \& Smith 2004).

\section{Conclusions}

Integral field spectroscopy permits simultaneous imaging over a wide range in wavelength. It can thus be used to reliably trace the excitation conditions across moderately-extended HerbigHaro objects without concern about the influence of changing atmospheric transmission or seeing between image frames. The main findings for HH 212 are as follows.

- The molecular hydrogen which produces the inner knots and bows of HH 212 is collisionally excited. The vibrational excitation temperatures are $\sim 2400 \mathrm{~K}$ for the inner knots NK 1 and SK 1 and $\sim 1700 \mathrm{~K}$ for the bows NB 1/2 and SB 1/2 observed further downwind.

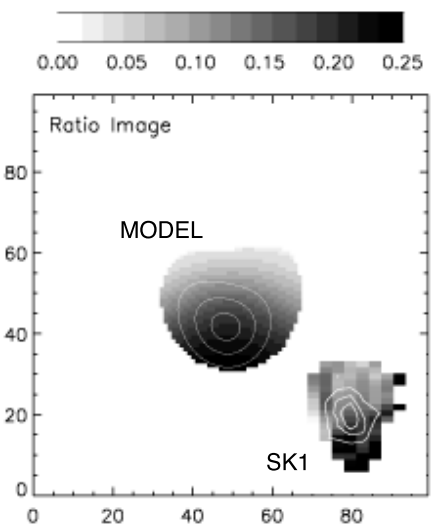

Fig. 14. The model (2-1 S(1)/1-0) S(1) ratio image is shown with overplotted contours corresponding to the model [Fe II] emission for comparison with the equivalent SK1 data extracted from Fig. 9.

- The $K$-band extinction found for the inner knots is $1.3 \pm$ 0.2 mag which corresponds to $\sim 12$ mag of visual extinction and a hydrogen column density of $\sim 2.2 \times 10^{22} \mathrm{~cm}^{-2}$. A much lower extinction is measured for the downwind bows of $<0.5 \mathrm{mag}$, suggesting that they have escaped from the higher density gas surrounding the outflow origin.

- Emission from [Fe II] at $1.644 \mu \mathrm{m}$ is detected alongside the $\mathrm{H}_{2}$ emission of the inner knots NK 1 and SK 1 . It is displaced along the jet direction relative to the $1-0 \mathrm{~S}(1)$ emission for both knots. This shows that higher excitation is located more distant from the driving source, as would be qualitatively consistent with a forward moving bow shock. However, the 


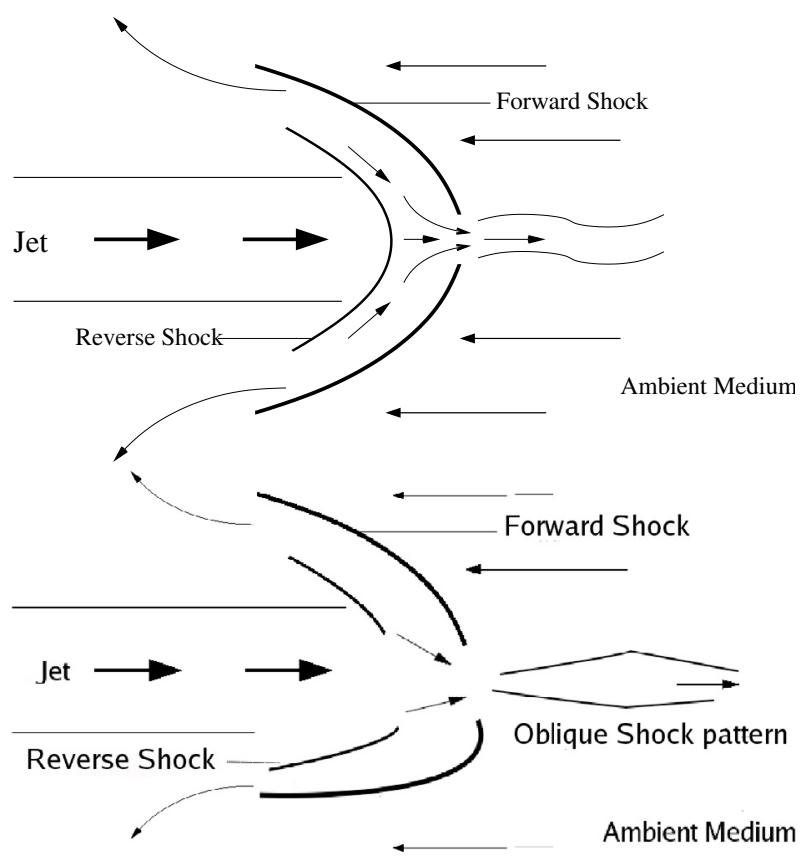

Fig. 15. An explanation for the inter-knot emission is provided by the the combined effect of a forward shock and a curved reverse shock. Whereas the ambient material is entrained and propelled outward by the forward shock, the jet shock entrains the jet material into a more collimated and focused flow which, under certain conditions such as high density, can breach ahead of the forward bow. As shown in the lower sketch, the knots can be asymmetric with oblique shocks exciting gas in the inter-knot region.

displacement was found to be small in comparison to the predictions of bow shock models. No [Fe II] was detected from the NB $1 / 2$ and SB $1 / 2$ bows.

- The positions of the peak fluxes depend on the upper energy level of the transitions for the knots. For SK 1 the peak positions from higher levels are more distant from the source, i.e. at the apex of the knot. The 2-1/1-0 S(1) flux ratio for SK 1 is also higher towards the south-western rim of the knot.

- The excitation for both NK 1 and SK 1 increases in a direction perpendicular to the outflow axis. The trend is in the same direction, from east to west, and occurs over the same spatial scale $\left(0.1^{\prime \prime}\right)$ for both knots. We note that the twin jets are not anti-parallel but display a small C-symmetry, bending to the west.

We have shown that the gradients in $\mathrm{H}_{2}$ excitation along the jet axis are generally consistent with bow shocks. In particular, we have modelled the inner knots NK 1 as J-type bow shocks and derived physical parameters. They correspond well to a bow of velocity $36 \mathrm{~km} \mathrm{~s}^{-1}$ propagating in a medium of density $9 \times$ $10^{4} \mathrm{~cm}^{-3}$. However, a faster bow $\left(76 \mathrm{~km} \mathrm{~s}^{-1}\right)$ with C-shock flanks would produce a very similar structure, as previously applied to bows in S233 (Khanzadyan et al. 2004). In both cases, an acute bow shock is favoured in order to reduce the iron emission from the bow cap and account for the moderate molecular excitation.

We have briefly considered mechanisms that could bring about a transverse distribution in excitation. Possibilities include (1) a relative drift of inner cloud material from west to east, (2) jet abrasion at the edges of an asymmetric outflow tunnel, (3) bow shock asymmetries due to the orientation of the magnetic field lines, and (4) a shear in the jet velocity. We find that option (3) is incapable of producing a significant transverse gradient.

A further indication of complex dynamics is the observed variations in the radial velocity across the knots (Davis et al. 2000). These are generally but not completely consistent with the observed direction of the radial velocity gradient in the circumstellar disk. As an alternative to rotation in the jet, the variation of order of a few $\mathrm{km} \mathrm{s}^{-1}$, could be due to a shear in the jet velocity. A jet flow at $5^{\circ}$ to the plane of the sky, the radial velocities then imply a shear along the jet axis in the range $10-30 \mathrm{~km} \mathrm{~s}^{-1}$. This would be sufficient to significantly displace the point of impact of the bow shocks transverse to the jet axis.

Therefore, a more intricate bow model is indicated. We suggest that, similar to the outer bows, the inner knots may also consist of two adjacent but distinct bow shocks. The bows are not perfectly aligned and the reverse inner bow deflects jet gas preferentially towards the west. The existence of oblique shocks also provides an interpretation for the narrow inter-knot $\mathrm{H}_{2}$ emission (see Fig. 15. Higher spatial and velocity resolution in the $1-0 \mathrm{~S}(1)$ line would determine if this is the shock configuration.

Acknowledgements. We wish to thank the Department of Culture, Arts and Leisure, Northern Ireland and INTAS grant 4838 for financial aid. The UK Infrared Telescope is operated by the Joint Astronomy Centre on behalf of the UK Particle Physics and Astronomy Research Council. Some of the data reported here were obtained as part of the UKIRT Service Programme. The remaining data were obtained during UIST commissioning: we acknowledge Suzanne Ramsay Howat, Stephen Todd and Sandy Leggett for their support during these observations. We also thank Ignacio Ugarte Urra for assistance with the images.

\section{References}

Beichman, C. A., Myers, P. C., Emerson, J. P., et al. 1986, ApJ, 307, 337

Caratti o Garatti, A., Giannini, T., Nisini, B., \& Lorenzetti, D. 2006, A\&A, 449, 1077

Davis, C. J., Berndsen, A., Smith, M. D., \& Chrysostomou, A. 2000, MNRAS, 314,241

Eislöffel, J., Smith, M. D., \& Davis, C. J. 2000, A\&A, 359, 1147

Fendt, C., \& Zinnecker, H. 1998, A\&A, 334, 750

Fendt, C., \& Zinnecker, H. 2000, in IAU Symp., 112

Froebrich, D. 2005, ApJS, 156, 169

Froebrich, D., Smith, M. D., Hodapp, K.-W., \& Eislöffel, J. 2003, MNRAS, 346, 163

Gibb, A. G., Richer, J. S., Chandler, C. J., \& Davis, C. J. 2004, ApJ, 603, 198

Khanzadyan, T., Smith, M. D., Davis, C. J., et al. 2003, MNRAS, 338, 57

Khanzadyan, T., Smith, M. D., Davis, C. J., \& Stanke, T. 2004, A\&A, 418, 163

Lee, C., Mundy, L. G., Reipurth, B., Ostriker, E. C., \& Stone, J. M. 2000, ApJ, 542,925

McCaughrean, M., Zinnecker, H., Andersen, M., Meeus, G., \& Lodieu, N. 2002, The Messenger, 109, 28

O’Connell, B., Smith, M. D., Davis, C. J., et al. 2004, A\&A, 419, 975

O'Connell, B., Smith, M. D., Froebrich, D., Davis, C. J., \& Eislöffel, J. 2005, A\&A, 431, 223

Reipurth, B., ed. 1989, Proceedings of the ESO Workshop on Low Mass Star Formation and Pre-main Sequence Objects, held in Garching bei Munchen, F.R.G., July 11-13, 1989

Rieke, G. H., \& Lebofsky, M. J. 1985, ApJ, 288, 618

Rosen, A., \& Smith, M. D. 2004, A\&A, 413, 593

Smith, M. D., \& Brand, P. W. J. L. 1990a, MNRAS, 242, 495

Smith, M. D., \& Brand, P. W. J. L. 1990b, MNRAS, 243, 498

Stanke, T. 2000, Ph.D. Thesis

Tedds, J. A., Smith, M. D., Fernandes, A. J. L., \& Davis, C. J. 2002, in Rev. Mex. Astron. Astrofis. Conf. Ser., 103

Völker, R., Smith, M. D., Suttner, G., \& Yorke, H. W. 1999, A\&A, 343, 953

Wiseman, J., Wooten, A., Zinnecker, H., \& McCaughrean, M. J. 2001, ApJ, 550, L87

Zinnecker, H., Bastien, P., Arcoragi, J. P., \& Yorke, H. W. 1992, A\&A, 265, 726 Zinnecker, H., McCaughrean, M. J., \& Rayner, J. 1996, Lecture Notes in Physics (Berlin: Springer Verlag), 465, 236

Zinnecker, H., McCaughrean, M. J., \& Rayner, J. T. 1998, Nature, 394, 862 
M. D. Smith et al.: Integral field spectroscopy of HH 212, Online Material p 1

\section{Online Material}


M. D. Smith et al.: Integral field spectroscopy of $\mathrm{HH} 212$, Online Material p 2

Table 1. Photometric results for NK 1 (top) and SK 1 (bottom). The upper energy level of the transition is given in the third column. The $x$ and $y$ peak position offsets are relative to the $1-0 \mathrm{~S}(1)$ peak position.

\begin{tabular}{lrrrrrr}
\hline \hline $\begin{array}{l}\text { NK 1 } \\
\text { line }\end{array}$ & $\begin{array}{r}\lambda \\
(\mu \mathrm{m})\end{array}$ & $\begin{array}{c}T \\
(\mathrm{~K})\end{array}$ & & & & \\
& & & & & & \\
\hline $\mathrm{Fe}[\mathrm{II}]$ & 1.644 & 11300 & 18.8 & 10.9 & -0.05 & 0.16 \\
$(1,0) \mathrm{S}(7)$ & 1.747 & 12817 & 16.1 & 10.5 & 0.18 & 0.12 \\
$(1,0) \mathrm{S}(6)$ & 1.788 & 11522 & 15.8 & 7.4 & 0.08 & -0.01 \\
$(1,0) \mathrm{S}(5)$ & 1.835 & 10341 & 40.5 & 32.2 & 0.06 & 0.10 \\
$(1,0) \mathrm{S}(4)$ & 1.891 & 9286 & 29.3 & 17.4 & 0.10 & 0.02 \\
$(1,0) \mathrm{S}(3)$ & 1.957 & 8365 & 137.0 & 65.2 & 0.06 & 0.06 \\
$(1,0) \mathrm{S}(2)$ & 2.033 & 7584 & 40.7 & 19.2 & 0.02 & 0.03 \\
$(2,1) \mathrm{S}(3)$ & 2.073 & 13890 & 12.9 & 8.1 & 0.12 & 0.05 \\
$(1,0) \mathrm{S}(1)$ & 2.121 & 6956 & 122.7 & 48.7 & 0.00 & 0.00 \\
$(2,1) \mathrm{S}(2)$ & 2.154 & 13150 & 5.2 & 3.9 & 0.06 & 0.10 \\
$(1,0) \mathrm{S}(0)$ & 2.223 & 6471 & 30.7 & 15.2 & -0.04 & 0.09 \\
$(2,1) \mathrm{S}(1)$ & 2.247 & 12550 & 12.5 & 7.3 & 0.10 & 0.08 \\
$(1,0) \mathrm{Q}(1)$ & 2.406 & 6149 & 112.3 & 47.4 & -0.07 & 0.07 \\
$(1,0) \mathrm{Q}(2)$ & 2.413 & 6471 & 30.9 & 16.3 & -0.04 & 0.05 \\
$(1,0) \mathrm{Q}(3)$ & 2.423 & 6956 & 109.6 & 45.4 & -0.07 & 0.02 \\
$(1,0) \mathrm{Q}(4)$ & 2.437 & 7584 & 31.8 & 17.2 & -0.05 & 0.07 \\
$(1,0) \mathrm{Q}(5)$ & 2.454 & 8365 & 43.0 & 20.8 & 0.11 & -0.05 \\
\hline
\end{tabular}

\begin{tabular}{lrrrrrr}
\hline SK 1 & $\begin{array}{r}\lambda \\
\text { line }\end{array}$ & $\begin{array}{c}T \text { total flux }{ }^{* *} \text { peak flux }{ }^{\dagger} \\
(\mathrm{K})\end{array}$ & $\begin{array}{r}x \text { offset } \\
\text { (arcsec) }\end{array}$ & $\begin{array}{r}y \text { offset } \\
(\operatorname{arcsec})\end{array}$ \\
\hline $\mathrm{Fe}[\mathrm{II}]$ & 1.644 & 11300 & 10.1 & 18.0 & 0.03 & -0.19 \\
$(1,0) \mathrm{S}(9)$ & 1.687 & 15722 & 3.1 & 5.6 & 0.27 & -0.31 \\
$(1,0) \mathrm{S}(8)$ & 1.714 & 14221 & 2.9 & 7.6 & 0.14 & -0.21 \\
$(1,0) \mathrm{S}(7)$ & 1.747 & 12817 & 23.4 & 23.4 & 0.06 & -0.08 \\
$(1,0) \mathrm{S}(6)$ & 1.788 & 11522 & 13.2 & 14.3 & 0.10 & -0.04 \\
$(1,0) \mathrm{S}(5)$ & 1.835 & 10341 & 38.7 & 39.1 & 0.03 & -0.06 \\
$(1,0) \mathrm{S}(4)$ & 1.891 & 9286 & 33.7 & 21.0 & 0.10 & -0.07 \\
$(1,0) \mathrm{S}(3)$ & 1.957 & 8365 & 145.9 & 101.2 & 0.05 & -0.01 \\
$(1,0) \mathrm{S}(2)$ & 2.033 & 7584 & 43.0 & 32.1 & 0.02 & -0.01 \\
$(2,1) \mathrm{S}(3)$ & 2.073 & 13890 & 22.2 & 21.8 & 0.05 & -0.09 \\
$(1,0) \mathrm{S}(1)$ & 2.121 & 6956 & 150.2 & 109.9 & 0.00 & 0.00 \\
$(2,1) \mathrm{S}(2)$ & 2.154 & 13150 & 6.4 & 8.2 & 0.11 & -0.07 \\
$(3,2) \mathrm{S}(3)$ & 2.201 & 19086 & 5.2 & 6.5 & 0.05 & -0.14 \\
$(1,0) \mathrm{S}(0)$ & 2.223 & 6471 & 30.3 & 22.9 & 0.01 & -0.02 \\
$(2,1) \mathrm{S}(1)$ & 2.247 & 12550 & 23.3 & 19.8 & 0.06 & -0.06 \\
$(2,1) \mathrm{S}(0)$ & 2.355 & 12095 & 4.2 & 6.3 & 0.00 & -0.15 \\
$(1,0) \mathrm{Q}(1)$ & 2.406 & 6149 & 134.8 & 87.7 & 0.01 & 0.00 \\
$(1,0) \mathrm{Q}(2)$ & 2.413 & 6471 & 42.4 & 30.6 & 0.02 & -0.02 \\
$(1,0) \mathrm{Q}(3)$ & 2.423 & 6956 & 137.9 & 96.2 & 0.01 & -0.02 \\
$(1,0) \mathrm{Q}(4)$ & 2.437 & 7584 & 42.5 & 34.1 & 0.02 & -0.03 \\
$(1,0) \mathrm{Q}(5)$ & 2.454 & 8365 & 85.3 & 64.9 & 0.02 & -0.01 \\
$(1,0) \mathrm{Q}(6)$ & 2.475 & 9286 & 29.1 & 23.7 & 0.07 & 0.00 \\
\hline & & & & & &
\end{tabular}

* Units of $10^{-18} \mathrm{~W} \mathrm{~m}^{-2}$. The $1 \sigma$ flux measurement uncertainty is $2.6 \times$ $10^{-18} \mathrm{~W} \mathrm{~m}^{-2}$ and $5.2 \times 10^{-18} \mathrm{~W} \mathrm{~m}^{-2}$ beyond $2.4 \mu \mathrm{m}$. This (standard deviation) value was derived from a large sample of flux measurements within apertures placed on sky regions and at various wavelengths. $* *$ Units of $10^{-18} \mathrm{~W} \mathrm{~m}^{-2}$. The $1 \sigma$ flux measurement uncertainty is $3.3 \times 10^{-18} \mathrm{~W} \mathrm{~m}^{-2}$ and $6.6 \times 10^{-18} \mathrm{~W} \mathrm{~m}^{-2}$ beyond $2.4 \mu \mathrm{m}$. $\dagger$ Units of $10^{-18} \mathrm{~W} \mathrm{~m}^{-2} \operatorname{arcsec}^{-2}$. The $1 \sigma$ noise level is $1.0 \times$ $10^{-18} \mathrm{~W} \mathrm{~m}^{-2} \operatorname{arcsec}^{-2}$.
Table 2. Photometric results for NB 1 and NB 2. The upper energy level of the transition is given in the third column. The $x$ and $y$ peak position offsets are relative to the $1-0 \mathrm{~S}(1)$ peak position.

\begin{tabular}{|c|c|c|c|c|c|c|}
\hline \multirow{2}{*}{$\begin{array}{l}\text { line } \\
\text { NB 1 }\end{array}$} & \multirow[t]{2}{*}{$\begin{array}{r}\lambda \\
(\mu \mathrm{m})\end{array}$} & \multicolumn{2}{|c|}{$\begin{array}{l}T \text { total flux * } \\
(\mathrm{K})\end{array}$} & \multirow[t]{2}{*}{ peak flux ${ }^{\dagger}$} & \multirow[t]{2}{*}{$\begin{array}{l}x \text { offset } \\
(\operatorname{arcsec})\end{array}$} & \multirow[t]{2}{*}{$\begin{array}{r}y \text { offset } \\
(\operatorname{arcsec})\end{array}$} \\
\hline & & & & & & \\
\hline$(1,0) \mathrm{S}(7)$ & 1.747 & 12817 & 12.9 & 5.7 & 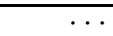 & \\
\hline$(1,0) \mathrm{S}(5)$ & 1.835 & 10341 & 58.2 & 15.8 & $\ldots$ & \\
\hline$(1,0) \mathrm{S}(4)$ & 1.891 & 9286 & 18.9 & 8.3 & $\cdots$ & $\ldots$ \\
\hline$(1,0) \mathrm{S}(3)$ & 1.957 & 8365 & 118.1 & 31.1 & 0.07 & 0.06 \\
\hline$(1,0) S(2)$ & 2.033 & 7584 & 33.3 & 7.9 & 0.0 & 0.04 \\
\hline$(1,0) \mathrm{S}(1)$ & 2.121 & 6956 & 88.2 & 23.4 & 0.00 & 0.00 \\
\hline$(1,0) \mathrm{S}(0)$ & 2.223 & 6471 & 13.7 & 6.0 & 0.13 & 0.05 \\
\hline$(2,1) \mathrm{S}(1)$ & 2.247 & 12550 & 4.4 & 2.5 & 0.14 & -0.13 \\
\hline$(1$, & 2.406 & 6149 & 51.9 & 20.9 & 0.08 & -0.03 \\
\hline (1 & 2.423 & 6956 & 57.2 & 22.0 & 0.06 & 0.00 \\
\hline$(1,0$ & 2.437 & 7584 & 7.3 & 5.7 & 0.06 & -0.16 \\
\hline$(1,0) \mathrm{Q}(5)$ & 2.454 & 8365 & 13.3 & 10.3 & $\cdots$ & \\
\hline \multicolumn{7}{|l|}{ NB 2} \\
\hline$(1,0) \mathrm{S}(7)$ & 1.747 & 12817 & 6.7 & 3.6 & $\cdots$ & \\
\hline$(1,0) \mathrm{S}(5)$ & 1.835 & 10341 & 40.9 & 13.8 & $\ldots$ & \\
\hline$(1,0) \mathrm{S}(4)$ & 1.891 & 9286 & 10.8 & 5.2 & $\ldots$ & $\ldots$ \\
\hline$(1,0) \mathrm{S}(3)$ & 1.957 & 8365 & 71.6 & 24.1 & -0.07 & 0.06 \\
\hline$(1,0) \mathrm{S}(2)$ & 2.033 & 7584 & 19.7 & 7.1 & -0.10 & 0.05 \\
\hline$(1,0) \mathrm{S}(1)$ & 2.121 & 6956 & 56.6 & 17.7 & 0.00 & 0.00 \\
\hline$(1,0) \mathrm{S}(0)$ & 2.223 & 6471 & 8.8 & 3.5 & $\cdots$ & \\
\hline$(2,1) \mathrm{S}(1)$ & 2.247 & 12550 & 2.4 & 2.2 & -0.16 & 0.18 \\
\hline$(1,0) \mathrm{Q}(1)$ & 2.406 & 6149 & 30.1 & 15.5 & 0.01 & 0.05 \\
\hline$(1,0) \mathrm{Q}(3)$ & 2.423 & 6956 & 31.7 & 16.8 & 0.01 & 0.00 \\
\hline$(1,0) \mathrm{Q}(4)$ & 2.437 & 7584 & 4 & 5.1 & 0.02 & 0.14 \\
\hline$(1,0) \mathrm{Q}(5)$ & 2.454 & 8365 & 3.1 & $\ldots$ & $\ldots$ & 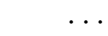 \\
\hline
\end{tabular}

* Units of Units of $10^{-18} \mathrm{~W} \mathrm{~m}^{-2}$. The $1 \sigma$ flux measurement uncertainty is $3.1 \times 10^{-18} \mathrm{~W} \mathrm{~m}^{-2}$. However, beyond $2.4 \mu \mathrm{m}$ this value increases to $6.2 \times 10^{-18} \mathrm{~W} \mathrm{~m}^{-2}$.

$\dagger$ Units of $10^{-18} \mathrm{~W} \mathrm{~m}^{-2} \operatorname{arcsec}^{-2}$. The $1 \sigma$ noise level is $1.6 \times$ $10^{-18} \mathrm{Wm}^{-2} \operatorname{arcsec}^{-2}$.

Table 3. Photometric results for SB 1/SB 2. The upper energy level of the transition is given in the third column. The $x$ and $y$ peak position offsets are relative to the $1-0 \mathrm{~S}(1)$ peak position.

\begin{tabular}{|c|c|c|c|c|c|c|}
\hline line & $\begin{array}{r}\lambda \\
(\mu \mathrm{m})\end{array}$ & $\begin{array}{r}T \\
(\mathrm{~K})\end{array}$ & 1 flux* & peak flux $^{\dagger}$ & $\begin{array}{c}x \text { offset } \\
(\operatorname{arcsec})\end{array}$ & $\begin{array}{r}y \text { offset } \\
(\operatorname{arcsec})\end{array}$ \\
\hline$(1,0) \mathrm{S}(7)$ & 1.747 & 12817 & 13.4 & 2.9 & -0.02 & -0.07 \\
\hline$(1,0) \mathrm{S}(3)$ & 1.957 & 8365 & 103.8 & 17.5 & -0.05 & 0.07 \\
\hline$(1,0) S(2)$ & 2.033 & 7584 & 28.1 & 4.9 & 0.04 & 0.08 \\
\hline$(2,1) \mathrm{S}(3)$ & 2.073 & 13890 & 10.1 & 2.8 & $\cdots$ & ... \\
\hline$(1,0) \mathrm{S}(1)$ & 2.121 & 6956 & 91.7 & 13.7 & 0.00 & 0.00 \\
\hline$(1,0) \mathrm{S}(0)$ & 2.223 & 6471 & 13 & 3.0 & $\cdots$ & $\cdots$ \\
\hline$(2,1) \mathrm{S}(1)$ & 2.247 & 12550 & 5.6 & 2.0 & . & . \\
\hline$(1,0) \mathrm{Q}(1)$ & 2.406 & 6149 & 58.4 & 14.1 & 0.04 & 0.08 \\
\hline$(1,0) \mathrm{Q}(3)$ & 2.423 & 6956 & 56.6 & 9.7 & 0.10 & 0.06 \\
\hline$(1,0) \mathrm{Q}(4)$ & 2.437 & 7584 & 13.4 & 5.3 & 0.08 & 0.05 \\
\hline$(1,0) \mathrm{Q}(5)$ & 2.454 & 8365 & 29.9 & 11.2 & . & .. \\
\hline$(1,0) \mathrm{Q}(6)$ & 2.475 & 9286 & 9.3 & 3.0 & $\cdots$ & $\cdots$ \\
\hline
\end{tabular}

* Units of Units of $10^{-18} \mathrm{~W} \mathrm{~m}^{-2}$. The $1 \sigma$ flux measurement uncertainty is $2.8 \times 10^{-18} \mathrm{~W} \mathrm{~m}^{-2}$ and $5.6 \times 10^{-18} \mathrm{~W} \mathrm{~m}^{-2}$ beyond $2.4 \mu \mathrm{m}$.

$\dagger$ Units of $10^{-18} \mathrm{Wm}^{-2} \operatorname{arcsec}^{-2}$. The $1 \sigma$ noise level is $1.0 \times$ $10^{-18} \mathrm{~W} \mathrm{~m}^{-2} \operatorname{arcsec}^{-2}$. 